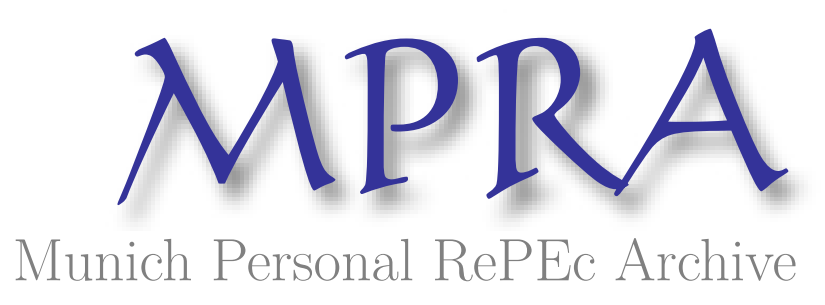

\title{
Globalization and Financial Market Contagion: Evidence from Financial Crisis and Natural Disasters
}

Asongu, Simplice

15 January 2013

Online at https://mpra.ub.uni-muenchen.de/56803/

MPRA Paper No. 56803, posted 23 Jun 2014 12:50 UTC 


\title{
AFRICAN GOVERNANCE AND DEVELOPMENT INSTITUTE
}

\section{$\underline{\text { A G D I Working Paper }}$}

\section{$\mathrm{WP} / 13 / 035$}

Globalization and Financial Market Contagion: Evidence from Financial Crisis and Natural Disasters

\author{
Simplice A. Asongu
}

African Governance and Development Institute,

P.O. Box 18 SOA/ 1365 Yaoundé, Cameroon.

E-mail: asongusimplice@yahoo.com 


\title{
AGDI Working Paper
}

Research Department

\section{Globalization and Financial Market Contagion: Evidence from Financial Crisis and Natural Disasters}

\author{
Simplice A. Asongu ${ }^{1}$
}

September 2013

\section{Overview}

With financial globalization, investors can gain from diversification if returns from financial markets are stable and not correlated. However with volatility spillovers, increase in crossmarket correlations exist as a real-effect and are not taken into account for asset allocation and portfolio composition. This chapter assesses financial contagion from two recent trends in the world economy: the global financial crisis and the 2011 Japanese natural disasters (tsunami, earthquake and nuclear crises).

\section{Introduction}

Globalization has been recognized as the principal force dominating the economic universe. It upholds to illuminate the world with economic prosperity and seeks a victory of market over government and self-interest over altruism. No less imperative is the global commitment to continuing and accelerating the pace of human development, which indicate the culmination of the historical processes of cultural advances. The dilemma however is that, while the phenomenon is a lusty, ineluctable process whose march can be stopped only by endangering the prosperity of peoples and nations, it also threatens to disfigure development in the manner it is evolving. As a dynamic force for change throughout the world, it is

\footnotetext{
${ }^{1}$ Simplice A. Asongu is Lead economist in the Research Department of the AGDI (asongus@afridev.org).
} 
expected to stimulate unprecedented surges in the wealth of nations by extending outwards the world production possibility frontier and redefining world markets as a "Global Village". With growing efforts toward financial liberalization, financial integration among economies has the benefit of improving allocation efficiency and diversifying risks. Despite these potential benefits, recent trends in the global economy suggest that crises in one country can easily spread to other countries through different channels as a result of financial globalization. In this chapter, we assess whether global financial markets have been vulnerable to contagion during some crises that have marked the global landscape in recent memory, notably: the 2007 global financial crisis and the 2011 Japanese tsunami, earthquake and nuclear crises. Hence, we aim to assess global evidence of financial market contagion from financial crisis and natural disasters.

Over the past decade, the concern about regional and global integration of emerging equity markets has been largely debated. The recent global financial meltdown and economic downturn has left many analysts concerned about whether emerging markets suffered from contagion (Asongu, 2012a). Most of these markets were still in their infancy before the start of the millennium, which rendered an examination of the transmission of financial variable movements from global crisis somewhat impractical. Hence, the effects of the US stock market crash of 1987, the Mexican peso crisis of 1994, Asian currency crisis of 1997, Russian and LTCM $^{2}$ crises of 1998, Brazilian crisis of 1999 and Turkish 2000/2001 crisis have unfortunately not been fully examined in all emerging equity markets. The recent financial crisis has provided a golden opportunity for such an investigation.

Natural disasters have inflicted serious damages on human life, property and economy (Asongu, 2012b). Though many earthquakes occur worldwide on a yearly basis and impact all walks of life in one way or the other, collateral effects resulting from such natural disasters could be quite detrimental financially and economically. The recent Japanese earthquake has resulted in collateral damages that make the disaster particularly significant. On March 11, 2011, Tohoku in Japan was hit by a 9.0 magnitude undersea mega thrust earthquake. This powerful shock triggered a tsunami that struck coastlines across the east of the country, leaving thousands dead and inflicting considerable property damages. But what has startled analysts and left them very concerned over the consequences of this earthquake is the nuclear disaster resulting there-from. Classified as a level-seven event on the International Nuclear Event Scale, the Fukushima nuclear incident has posed a risk equal to the worst nuclear power

\footnotetext{
${ }^{2}$ Long-term Capital Management.
} 
plant accident in history (Chernobyl disaster). With much uncertainty over how the crisis would have been managed, it is imperative to investigate how international financial markets reacted three months into the crises. Therefore, the second goal of this chapter is to examine whether any contagion effect occurred a few months after the Japanese earthquake, tsunami and worst nuclear crisis since Chernobyl.

There are many reasons this chapter should be dedicated to studying the extent to which financial markets have been affected by the recent financial and natural crises. Among others, results of the studies could enable analysts and policy makers evaluate the benefits of international trade and cross-border investments, and hence, the attractiveness of foreign capital inflows. The results could also provide some basis on how developing countries stand to benefit (loss) from (in) long-run investment sources and global financial booms (as a result of external financial shocks) through financial market integration. The rest of the chapter is organized as follows. Section 2 examine related literature with emphasis on the effects of financial market integration, linkages between financial market integration and crises, definitions and channels of contagion and, how to measure contagion. Section 3 provides evidence of contagion from the 2007 global financial crisis in financial markets of developing countries. In Section 4, global international stock indices and exchange rates are investigated for contagion after the 2011 Japanese tsunami, earthquake and nuclear crises. We conclude with Section 5.

\section{Related literature}

\subsection{Effects of financial market integration}

Financial integration between economies is believed to have two main positive impacts: the improvement of capital allocation efficiency and diversification of risks (Demyanyk and Volosovych, 2008; Coulibaly, 2009; Kose et al., 2011; Asongu, 2012ab). However, the recent global financial crisis which is considered by many analysts and policymakers as the worst since the Great Depression has cast a dark shadow on the contagion effect of financial integration; despite its advantages. There is a broad economics and finance literature that addresses the potential benefits of financial integration.

From a theoretical perspective, financial globalization should facilitate efficient international allocation of capital and improve international risks sharing (Kose et al., 2011; Asongu, 2012a). Asongu (2012a) posit that, the benefits are much greater for developing countries because they are relatively scarce in capital and rich in labor sources. Accordingly, access to foreign capital should help them grow faster through new sources of investment. He 
has further professed that, since developing countries have more volatile output growth than advanced industrial economies, their potential welfare gains from international risk sharing are much greater. The findings of Kose et al. (2011) reveal that, with certain identifiable thresholds in variables such as financial depth and institutional quality, the cost-benefit tradeoff from financial openness improves significantly once the threshold conditions are met. Demyanyk and Volosovych (2008) much earlier had analyzed the benefits of financial integration (resulting from international risk sharing) among 25 European Union (EU) countries and presented a case for diversification of risk across EU member states if the risks are fully shared. In a nutshell they emphasized that; the 10 new members joining the EU would have higher gains than the long standing 15 members. The most glaring indication of financial integration benefits is the case of South Africa, a country that has experienced financial autarky as a result of the embargo imposed in 1985 and removed in 1993. Consistent with Coulibaly (2009), there was a significant decrease in the rates of investment, capital and output during the embargo period in South Africa in comparison with the pre-embargo and post-embargo periods.

During the embargo South Africa might have benefited from financial isolation in event of a global financial meltdown. This implies, countries in relative financial autarky are less vulnerable to international financial shocks. Though one of the main appeals of financial integration is risk diversification, paradoxically, increased financial globalization can reduce the scope for risk diversification because integrated markets tend to be more interdependent and highly correlated. Another downside of financial integration could be linked to threshold factors pointed-out earlier by Kose et al. (2011). Their study has revealed that, countries with low levels of financial depth and institutional quality do not stand to benefit from financial integration. This perspective is in line with Schmukler (2004) who had stressed that: the importance of sound financial fundamentals and strong macroeconomic institutions; the presence of which should enable more effective management of crises and lower the probability of crises and contagion. Hence, financial globalization could itself be a source of crises.

\subsection{Linkages between financial integration (globalization) and crises}

We have observed that financial globalization has several potential benefits. However the recent stream of financial crises and contagion due to the growing liberalization of financial systems and integration of financial markets around the world, have led many analysts to conclude that globalization breeds financial volatility and crises (Asongu, 2012ab). 
Though domestic factors are usually at the origin of crises, there are different channels through which financial globalization could be related to crises.

Firstly, as emphasized by Schmukler (2004), when a country's financial system is liberalized, it becomes an object of market discipline exercised by both foreign and domestic investors. In a closed economy, only domestic investors monitor and react to unsound economic fundamentals while, in an open one domestic and foreign investors might prompt the country to achieve sound fundamentals. As pointed-out earlier, the absence of sound macroeconomic, financial and institutional fundamentals could substantially increase the probability of crises. It logically follows that, conflicting interests and views between investors (domestic and foreign) on key fundamentals might precipitate crises and reduce the ability of governments to effectively monitor and manage them.

Secondly, even with sound domestic economic fundamentals and quality institutions, international financial market imperfections could also lead to crises. Among other things, these could lead to irrational behavior, herding behavior, speculative attacks, bubbles, and crashes. In plainer terms, regardless of market fundamentals, investors could speculate against a currency if they believe that the exchange rate is unsustainable, which could ultimately lead to self-fulfilling balance-of-payments. This thesis illustrated by Obstfeld (1986) has been purported by Schmukler (2004), amongst others.

Thirdly, even in the presence of sound fundamentals and absence of imperfections in international capital markets, crises might still arise on the grounds of external factors (Schmukler, 2004) such as determinants of capital flows (Calvo et al., 1996) and foreign interest rates (Frankel and Rose, 1996). For example if a country becomes dependent on foreign capital, variations in foreign capital flows could create financial issues and economic downturns. Frankel and Rose (1996) clearly emphasize the role foreign interest rates play in determining the likelihood of financial crises in developing countries.

Fourthly, still consistent with Schmukler (2004), financial globalization could lead to financial crises by contagion, namely by shocks via real links, financial links and herdingbehavior or unexplained high correlations. This chapter will focus on this fourth example ${ }^{3}$; the elucidation and definition of which are worthwhile.

\subsection{Definitions and channels of contagion}

\subsubsection{Definitions of contagion}

\footnotetext{
${ }^{3}$ That on the link between financial integration and crisis.
} 
There is no consensus on the definition of contagion among economists. According to the World Bank, the phenomenon can be defined from three angles. Firstly, from a broad angle, contagion could be identified with the general process of stock transmission across countries. Thus, it is worthwhile understanding that this definition does encompass both negative shocks and positive spillover effects. Secondly, the phenomenon could be conceived as the propagation of shocks between two countries in excess of what should be expected, based on the fundamentals after considering co-movements triggered by common shocks. This second definition is restricted only to shocks and presupposes the mastery of what constitutes the underlying fundamentals (without which an appraisal of excess co-movements is not possible). The last and more restrictive definition considers the phenomenon as the change in the transmission mechanisms (channels) that take place during a period of turmoil and is appreciated by a significant increase in cross-market correlations. Within the framework of this chapter, we shall be restricted to the third definition because: (1) our studies aim to investigate recent trends in some crises of global dimension which are negative shocks and not positive spillovers (as opposed to the first definition) and; (2) we do not master what constitutes underlying fundamentals of co-movements we are about to study (in antagonism to the second definition).

From an empirical standpoint, the third definition was first proposed by Forbes and Rigobon (2002). They assessed contagion as a significant increase in market co-movements after a shock has occurred in one country (or market). According to this definition, the condition for contagion is a significant increase in co-movements as a result of a shock in one market. Accordingly, if two markets display a high degree of co-movements during the stability period, even if they are highly correlated during a crisis, if this crisis-correlation is not significant it does not amount to contagion. In the absence of a significant correlation during the crisis-period, the term 'interdependence' is used to qualify the situation between the two markets (or countries).

\subsubsection{Channels of contagion}

Borrowing from Schmukler (2004), three mains channels of contagion have been documented in the literature. (1) Firstly, real links which are often associated with trading activities. For example if two countries are trading partners and compete in the same external market, a devaluation of the exchange rate of one country deteriorates the other country's competitive advantage. In order to rebalance its external sectors, the losing country would 
want to devaluate its own currency. The Sino-American trade relation today is an eloquent example. (2) Secondly, a financial link is a situation where two economies are connected through the international financial system. For instance, let us consider leverage institutions facing margin calls. In case the value of the collateral falls as a result of a negative shock in one country, in a bid to increase their initial stock, these institutions would sell some of their holdings in countries not yet affected by the shock. This gives rise to a mechanism that ripples the shocks to other countries. (3) Thirdly, due to herding behaviors or panics resulting from asymmetric information, financial markets might transmit shocks across markets. For obvious reasons (common sense), we shall not elaborate on the mechanics of this third type.

\subsection{Measuring contagion}

Many methods of measuring contagion have been documented in the literature to appreciate the spreading of international shocks across countries. The most widely employed are cross-market correlation coefficients procedures (King and Wadhwani, 1990; Forbes and Rigobon, 2002; Collins and Biekpe, 2003; Lee et al., 2007; Asongu, 2011; Asongu, 2012ab), cross-market co-integration vectors changing techniques (Kanas, 1998), volatility analysis based on ARCH and GARCH models (King et al., 1994) and direct estimation of specific transmission mechanisms (Forbes, 2000). Consistent with the restrictive definition of contagion, the chapter shall adopt Forbes and Rigobon (2002) in the context of: (1) Collins and Biekpe $(2003)^{4}$ for the assessment of financial (Section 3) and political (Section 5) crises and; (2) Lee et al. (2007) for the examination of natural disasters (Section 4).

\section{The 2007 financial crisis}

Financial integration among economies has the benefit of improving allocation efficiency and diversifying risk. However the recent global financial crisis, considered as the worst since the Great Depression has re-ignited the fierce debate about the merits of financial globalization and its implications for growth especially in developing countries (Asongu, 2012a). This section examines whether equity markets in emerging countries were vulnerable to contagion during the recent global financial meltdown.

\subsection{Data}

\footnotetext{
${ }^{4}$ The hypothesis testing in Collins and Biekpe (2003) is slightly different from that of Forbes and Rigobon (2002) in that, the test statistics to determine contagion is not calculated using estimated sample variances. Their test statistics (Collins and Biekpe, 2003) uses exact student statistics based on actual sample correlation coefficients (Asongu, 2012a). Contagion is then measured by the significant increase in adjusted correlation coefficients during the crisis period as compared with the stable period.
} 
The purpose of this study is to investigate correlations between the returns of the USA stock index and stock indices of emerging countries. With the Dow Jones Industrial Average as the 'base criterion', we analyze if co-movements between the 'base criterion' and financial markets of developing countries were significantly strengthened during the recent global financial crisis. In et al. (2008), MacAndrews (2008), Taylor and William (2008) and more recently Ji and In (2010) have all used the August $9^{\text {th }} 2007$ date as the start of the financial crisis $^{5}$. The sample period is divided into two categories: a 14 month pre-crisis period also known as the tranquil or stable period and, a 15 month crisis or turmoil period. In a bid to make our findings robust, the turmoil period is further divided into three sections ${ }^{6}$ : the shortrun or four month crisis-period (August 09, 2007 to December 06, 2007); the medium-term or eight months crisis-period (August 09, 2007 to April 10, 2008) and the long-term or 15 month crisis-period (August 09, 2007 to November 13, 2008). Weekly data used in the study is obtained from Bloomberg's database. We use the local currency index return because Forbes and Rigobon (2002) have shown that using dollar or local indices will produce similar outcomes.

\subsection{Methodology}

Contagion is defined by Forbes and Rigobon as a significant increase in market comovements after a shock has occurred in one country ${ }^{7}$.

The correlation coefficient is defined as:

$$
\rho=\frac{\sigma_{x y}}{\sigma_{x} \sigma_{y}}
$$

where: ' $x$ ' is the base criterion while ' $y$ ' is an emerging equity market.

Consistent with Forbes and Rigobon, the correlation coefficient is adjusted in the following manner:

$$
\rho^{*}=\frac{\rho}{\left.\sqrt{1+\delta\left[1-(\rho)^{2}\right.}\right]}
$$

\section{Where:}

\footnotetext{
${ }^{5}$ Date at which, BNP Paribas announced the closure of its funds that held US subprime debts.

${ }^{6}$ From the empirical literature, the tranquil period is always longer than the turmoil period. For instance it is longer by a year, ten and a half months and nine months in Forbes \& Rigobon (2002), Collins \& Biekpe (2003) and Lee et al. (2007) respectively.

${ }^{7}$ According to this definition, the presence of high correlation between two markets during the stable period and eventually a sustained increase in the high degree of cross market co-movements at the turmoil period does not amount to contagion. Hence, contagion according to this definition is the presence of significant increase in comovements after a shock. On the other hand, if the high correlation degree is not significant, the term 'interdependence' is used to qualify to relationship.
} 
$\delta=\frac{\sigma_{x x}^{h}}{\sigma_{x x}^{l}}-1$

which measures the change in high-period volatility against low-period volatility. The crisisperiod is used as the 'high volatility period' and the tranquil period as the 'low volatility period' in the calculation of the correlation coefficient adjuster. Contagion is subsequently measured as the significance of adjusted correlation coefficients in time-varying turmoil periods versus the stability period.

In the empirical literature, Collins and Biekpe (2003) and Lee et al. (2007) have respectively applied both the t-test and F-test for the significance of the difference in correlations. Accordingly, when only one coefficient is to be estimated, both tests have the same implications (Asongu, 2011; Asongu, 2012ab). Consistent with the t-statistics approach, the significance of increase in correlations during the turmoil period (t) with respect to the stable(s) period is defined by:

$t=\left(\rho_{t}-\rho_{s}\right) \sqrt{\frac{n_{t}+n_{s}-4}{1-\left(\rho_{t}-\rho_{s}\right)^{2}}}$

Where

$$
t_{\left(0.01, n_{t}+n_{s}-4\right)}
$$

with, $n \mathrm{t}(n \mathrm{~s})$ indicating actual observed weeks during the turmoil (stable) period.

The following hypotheses are then tested:

$H_{o}: \rho_{1}-\rho_{2}=0$ versus $H_{1}: \rho_{1}-\rho_{2}>0$

Where $H_{o}$ is the null hypothesis of no contagion while $H_{1}$ is the alternative hypothesis for the presence of contagion 
Table1: International stock indexes returns conditional (unadjusted) correlation coefficient $\mathrm{s}$ in 2007 financial crisis

\begin{tabular}{|c|c|c|c|c|c|c|c|c|c|c|c|c|c|c|c|c|c|}
\hline \multirow[t]{2}{*}{ Regions } & \multirow[t]{2}{*}{ Countries } & \multicolumn{2}{|c|}{ Full period } & \multicolumn{2}{|c|}{ Stable period } & \multicolumn{3}{|c|}{ Short-term turmoil period } & \multirow[b]{2}{*}{ Co } & \multicolumn{3}{|c|}{ Medium-term turmoil period } & \multicolumn{4}{|c|}{ Long-term turmoil period } & \multirow[b]{2}{*}{ Co } \\
\hline & & $\rho$ & $\sigma$ & $\rho$ & $\sigma$ & $\rho$ & $\sigma$ & t-test & & $\rho$ & $\sigma$ & t-test & Co & $\rho$ & $\sigma$ & t-test & \\
\hline \multirow{9}{*}{ Africa } & Botswana & -0.040 & 0.015 & 0.024 & 0.014 & 0.573 & 0.010 & $5.641 * * *$ & $\mathrm{Y}$ & 0.197 & 0.008 & $1.675 *$ & $\mathrm{Y}$ & -0.188 & 0.013 & $-2.419 * *$ & $\mathrm{~N}$ \\
\hline & Egypt & 0.336 & 0.045 & 0.196 & 0.034 & 0.419 & 0.028 & $1.968 *$ & $\mathrm{Y}$ & 0.212 & 0.028 & 0.154 & $\mathrm{~N}$ & 0.353 & 0.051 & $1.757 *$ & $\mathrm{Y}$ \\
\hline & Kenya & 0.083 & 0.034 & 0.008 & 0.028 & 0.049 & 0.030 & 0.494 & $\mathrm{~N}$ & -0.178 & 0.038 & -1.656 & $\mathrm{~N}$ & 0.079 & 0.038 & 0.970 & $\mathrm{~N}$ \\
\hline & Mauritius & 0.302 & 0.030 & 0.003 & 0.028 & 0.001 & 0.024 & 0.039 & $\mathrm{~N}$ & -0.099 & 0.027 & -0.922 & $\mathrm{~N}$ & 0.382 & 0.031 & $4.636 * * *$ & $\mathrm{Y}$ \\
\hline & Morocco & 0.059 & 0.024 & 0.024 & 0.025 & 0.022 & 0.019 & -0.014 & $\mathrm{~N}$ & -0.109 & 0.019 & -1.288 & $\mathrm{~N}$ & 0.051 & 0.021 & 0.294 & $\mathrm{~N}$ \\
\hline & Namibia & 0.376 & 0.037 & 0.417 & 0.024 & 0.558 & 0.034 & 1.219 & $\mathrm{~N}$ & 0.111 & 0.043 & $-3.093 * * *$ & $\mathrm{~N}$ & 0.342 & 0.045 & -0.845 & $\mathrm{~N}$ \\
\hline & Nigeria & 0.027 & 0.038 & 0.095 & 0.032 & -0.457 & 0.027 & $-5.710^{* * * *}$ & $\mathrm{~N}$ & -0.410 & 0.026 & $-5.617 * * *$ & $\mathrm{~N}$ & -0.060 & 0.040 & $-1.743^{*}$ & $\mathrm{~N}$ \\
\hline & South A & 0.435 & 0.030 & 0.380 & 0.021 & 0.674 & 0.024 & $2.641^{* *}$ & $\mathrm{Y}$ & 0.238 & 0.031 & -1.378 & $\mathrm{~N}$ & 0.428 & 0.036 & 0.522 & $\mathrm{~N}$ \\
\hline & Tunisia & 0.258 & 0.016 & 0.129 & 0.014 & 0.183 & 0.009 & 0.462 & $\mathrm{~N}$ & 0.165 & 0.018 & 0.343 & $\mathrm{~N}$ & 0.341 & 0.018 & $2.405^{* * *}$ & $\mathrm{Y}$ \\
\hline \multirow{8}{*}{$\begin{array}{l}\text { Middle } \\
\text { East }\end{array}$} & A Dhabi & -0.069 & 0.030 & -0.053 & 0.021 & 0.246 & 0.024 & $2.706 * * *$ & $\mathrm{Y}$ & -0.133 & 0.025 & -0.761 & $\mathrm{~N}$ & -0.086 & 0.037 & -0.356 & $\mathrm{~N}$ \\
\hline & Bahrain & 0.017 & 0.015 & -0.031 & 0.013 & 0.477 & 0.013 & $5.069 * * *$ & $\mathrm{Y}$ & 0.173 & 0.012 & $1.998 * *$ & Y & -0.004 & 0.017 & 0.297 & $\mathrm{~N}$ \\
\hline & Dubai & -0.085 & 0.039 & -0.027 & 0.027 & -0.160 & 0.031 & -1.146 & $\mathrm{~N}$ & -0.173 & 0.030 & -1.410 & $\mathrm{~N}$ & -0.126 & 0.048 & -1.089 & $\mathrm{~N}$ \\
\hline & Israel & 0.264 & 0.028 & 0.531 & 0.023 & 0.697 & 0.019 & 1.444 & $\mathrm{~N}$ & 0.287 & 0.025 & $-2.411 * *$ & $\mathrm{~N}$ & 0.089 & 0.032 & $-5.462 * * *$ & $\mathrm{~N}$ \\
\hline & Jordan & 0.015 & 0.031 & 0.044 & 0.020 & 0.148 & 0.016 & 0.893 & $\mathrm{~N}$ & 0.034 & 0.020 & -0.105 & $\mathrm{~N}$ & 0.011 & 0.040 & -0.381 & $\mathrm{~N}$ \\
\hline & Kuwait & -0.085 & 0.026 & n.a & n.a & 0.681 & 0.014 & n.a & & 0.106 & 0.013 & n.a & & -0.085 & 0.026 & n.a & \\
\hline & Lebanon & 0.200 & 0.033 & 0.226 & 0.023 & 0.145 & 0.023 & -0.710 & $\mathrm{~N}$ & 0.181 & 0.021 & -0.441 & $\mathrm{~N}$ & 0.213 & 0.040 & -0.155 & $\mathrm{~N}$ \\
\hline & Oman & -0.217 & 0.031 & 0.112 & 0.016 & 0.013 & 0.019 & -0.865 & $\mathrm{~N}$ & -0.261 & 0.028 & $-3.867 * * *$ & $\mathrm{~N}$ & -0.306 & 0.040 & $-5.112 * * *$ & $\mathrm{~N}$ \\
\hline \multirow{15}{*}{ Asia } & Qatar & -0.133 & 0.040 & -0.032 & 0.030 & 0.186 & 0.027 & $1.930 *$ & $\mathrm{Y}$ & -0.101 & 0.037 & -0.653 & $\mathrm{~N}$ & -0.175 & 0.047 & -1.595 & $\mathrm{~N}$ \\
\hline & Saudi A & 0.012 & 0.047 & 0.059 & 0.041 & -0.302 & 0.027 & $-3.339 * * *$ & $\mathrm{~N}$ & -0.113 & 0.053 & $-1.681 *$ & $\mathrm{~N}$ & -0.002 & 0.052 & 0.522 & $\mathrm{~N}$ \\
\hline & China & 0.073 & 0.056 & 0.071 & 0.048 & 0.528 & 0.045 & $4.507 * * *$ & $\mathrm{Y}$ & 0.071 & 0.048 & 0.064 & $\mathrm{~N}$ & 0.063 & 0.012 & -0.582 & $\mathrm{~N}$ \\
\hline & Dhaka & 0.047 & 0.024 & -0.275 & 0.020 & -0.462 & 0.022 & $-6.698 * * *$ & $\mathrm{~N}$ & -0.275 & 0.020 & $-4.539 * * *$ & $\mathrm{~N}$ & -0.132 & 0.020 & $-3.289 * * *$ & $\mathrm{Y}$ \\
\hline & India & 0.264 & 0.038 & 0.252 & 0.044 & 0.400 & 0.042 & 0.574 & $\mathrm{~N}$ & 0.252 & 0.044 & -0.778 & $\mathrm{~N}$ & 0.212 & 0.048 & -1.355 & $\mathrm{~N}$ \\
\hline & Indonesia & 0.057 & 0.040 & 0.394 & 0.054 & 0.773 & 0.055 & $5.268 * * *$ & $\mathrm{Y}$ & 0.394 & 0.054 & 1.389 & $\mathrm{~N}$ & -0.031 & 0.052 & $-3.263 * * *$ & $\mathrm{~N}$ \\
\hline & Malaysia & 0.100 & 0.026 & 0.457 & 0.036 & 0.838 & 0.034 & $6.045 * * *$ & $\mathrm{Y}$ & 0.457 & 0.036 & $1.903^{*}$ & $\mathrm{Y}$ & 0.015 & 0.031 & $-2.832 * * *$ & $\mathrm{~N}$ \\
\hline & Mongolia & 0.062 & 0.046 & -0.093 & 0.044 & -0.175 & 0.056 & 0.665 & $\mathrm{~N}$ & -0.093 & 0.044 & 1.538 & $\mathrm{~N}$ & 0.049 & 0.038 & $3.499 * * *$ & $\mathrm{Y}$ \\
\hline & Pakistan & 0.021 & 0.037 & 0.330 & 0.028 & 0.338 & 0.033 & $2.584 * *$ & $\mathrm{Y}$ & 0.330 & 0.028 & $2.798 * * *$ & $\mathrm{Y}$ & -0.031 & 0.042 & -0.898 & $\mathrm{~N}$ \\
\hline & Philippines & 0.361 & 0.040 & 0.621 & 0.045 & 0.855 & 0.053 & $7.127 * * *$ & $\mathrm{Y}$ & 0.621 & 0.045 & $4.229 * * *$ & $\mathrm{Y}$ & 0.373 & 0.048 & $1.749 *$ & $\mathrm{Y}$ \\
\hline & S. Korea & 0.469 & 0.034 & 0.640 & 0.041 & 0.822 & 0.047 & $10.324 * * *$ & $\mathrm{Y}$ & 0.640 & 0.041 & $6.945 * * *$ & $\mathrm{Y}$ & 0.502 & 0.042 & $\mathbf{5 . 5 6 2} * * *$ & $\mathrm{Y}$ \\
\hline & Sri Lanka & 0.204 & 0.027 & 0.380 & 0.019 & -0.100 & 0.021 & -0.828 & $\mathrm{~N}$ & 0.380 & 0.019 & $3.997 * * *$ & $\mathrm{Y}$ & 0.288 & 0.027 & $3.390 * * *$ & $\mathrm{Y}$ \\
\hline & Taiwan & 0.429 & 0.035 & 0.415 & 0.040 & 0.836 & 0.041 & $18.401 * * *$ & $\mathrm{Y}$ & 0.415 & 0.040 & $5.315 * * *$ & $\mathrm{Y}$ & 0.482 & 0.043 & 7.331*** & $\mathrm{Y}$ \\
\hline & Thailand & 0.355 & 0.037 & 0.422 & 0.039 & 0.715 & 0.035 & $5.908 * * *$ & $\mathrm{Y}$ & 0.422 & 0.039 & $2.722 * * *$ & $\mathrm{Y}$ & 0.385 & 0.046 & $2.698 * * *$ & $\mathrm{Y}$ \\
\hline & Vietnam & 0.204 & 0.060 & 0.319 & 0.056 & 0.524 & 0.032 & $3.842 * * *$ & $\mathrm{Y}$ & 0.319 & 0.056 & $1.985^{*}$ & $\mathrm{Y}$ & 0.195 & 0.068 & 0.876 & $\mathrm{~N}$ \\
\hline \multirow{9}{*}{$\begin{array}{l}\text { Latin } \\
\text { America }\end{array}$} & Argentina & 0.543 & 0.041 & 0.644 & 0.026 & 0.752 & 0.045 & 0.934 & $\mathrm{~N}$ & 0.630 & 0.037 & -0.136 & $\mathrm{~N}$ & 0.505 & 0.051 & -1.556 & $\mathrm{~N}$ \\
\hline & Brazil & 0.773 & 0.043 & 0.797 & 0.027 & 0.831 & 0.043 & 0.290 & $\mathrm{~N}$ & 0.720 & 0.042 & -0.744 & $\mathrm{~N}$ & 0.765 & 0.052 & -0.358 & $\mathrm{~N}$ \\
\hline & Chile & 0.690 & 0.034 & 0.588 & 0.020 & 0.721 & 0.040 & 1.154 & $\mathrm{~N}$ & 0.710 & 0.040 & 1.178 & $\mathrm{~N}$ & 0.703 & 0.043 & 1.281 & $\mathrm{~N}$ \\
\hline & Columbia & 0.475 & 0.032 & 0.336 & 0.026 & 0.381 & 0.030 & 0.386 & $\mathrm{~N}$ & 0.616 & 0.034 & $2.802 * * *$ & $\mathrm{Y}$ & 0.504 & 0.036 & $1.896 *$ & $\mathrm{Y}$ \\
\hline & Costa Rica & -0.020 & 0.028 & -0.085 & 0.031 & -0.088 & 0.019 & -0.025 & $\mathrm{~N}$ & -0.203 & 0.023 & -1.140 & $\mathrm{~N}$ & -0.083 & 0.021 & 0.023 & $\mathrm{~N}$ \\
\hline & Ecuador & 0.030 & 0.029 & 0.085 & 0.015 & 0.010 & 0.005 & -0.648 & $\mathrm{~N}$ & 0.040 & 0.049 & -0.431 & $\mathrm{~N}$ & 0.016 & 0.037 & -0.773 & $\mathrm{~N}$ \\
\hline & Mexico & 0.774 & 0.037 & 0.721 & 0.026 & 0.814 & 0.037 & 0.800 & $\mathrm{~N}$ & 0.865 & 0.037 & 1.391 & $\mathrm{~N}$ & 0.784 & 0.044 & 0.692 & $\mathrm{~N}$ \\
\hline & Peru & 0.422 & 0.052 & -0.066 & 0.029 & 0.907 & 0.063 & $35.962 * * *$ & $\mathrm{Y}$ & 0.693 & 0.059 & $11.16 * * *$ & $\mathrm{Y}$ & 0.478 & 0.065 & $7.185 * * *$ & Y \\
\hline & Venezuela & 0.119 & 0.034 & 0.035 & 0.038 & 0.193 & 0.027 & 1.379 & $\mathrm{~N}$ & 0.269 & 0.034 & $2.313 * *$ & $\mathrm{Y}$ & 0.159 & 0.030 & 1.385 & $\mathrm{~N}$ \\
\hline
\end{tabular}

The table shows the conditional (unadjusted) cross market correlation coefficients ( $\rho$ ) and standard deviations for the US and other stock markets. Test statistics is obtained from t-transformations. The stable period is defined as the 14-month pre-crisis period (June 08, 2006 to August 09, 2007). The short-term turmoil period is defined as the four-month crisis period (August 09, 2007 to December 06, 2007). The medium-term turmoil period is defined as the eight months crisis period (August 09, 2007 to April 10, 2008). The long-term turmoil period is defined the fifteen months crisis period (August 09, 2007 to November 13, 2008). The full period is the stable period plus the long-term turmoil period (June 08, 2006 to November 13, 2008). Contagion (Co) occurs (Y)when the test statistics is greater than the critical values. No contagion (N) occurs when the test statistics is less than or equal to the critical value.*, **,***: represent significance at 10\%, 5\% and 1\% respectively. (nt+ns-4) degrees of freedom for the t-statistics are (66+61-4); (35+61-4);(17+61-4) for the long, medium and short terms respectively. $\sigma$ : represents the standard deviation. 
Table 2: International stock indexes returns unconditional (adjusted) correlation coefficient in 2007 financial crisis

\begin{tabular}{|c|c|c|c|c|c|c|c|c|c|c|c|c|c|c|c|c|c|c|}
\hline \multirow[t]{2}{*}{ Regions } & \multirow[t]{2}{*}{ Countries } & \multicolumn{2}{|c|}{ Full period } & \multicolumn{2}{|c|}{ Stable period } & \multicolumn{5}{|c|}{ Short-term turmoil period } & \multicolumn{4}{|c|}{ Medium-term turmoil period } & \multicolumn{4}{|c|}{ Long-term turmoil period } \\
\hline & & $\rho$ & $\sigma$ & $\rho^{*}$ stp & $\rho^{*} \mathrm{mtp}$ & $\rho^{*} \operatorname{ltp}$ & $\rho^{*}$ & $\delta$ & t-test & Co & $\rho^{*}$ & $\delta$ & t-test & Co & $\rho^{*}$ & $\delta$ & t-test & $\mathrm{Co}$ \\
\hline \multirow{9}{*}{ Africa } & Botswana & -0.040 & 0.015 & 0.030 & 0.034 & 0.026 & 0.647 & -0.321 & $6.747 * * *$ & Y & 0.265 & -0.466 & $2.278 * *$ & Y & -0.197 & -0.090 & $-2.538 * *$ & $\mathrm{~N}$ \\
\hline & Egypt & 0.336 & 0.045 & 0.219 & 0.217 & 0.163 & 0.459 & -0.202 & $2.133 * *$ & $\mathrm{Y}$ & 0.234 & -0.189 & 0.168 & $\mathrm{~N}$ & 0.296 & 0.475 & 1.498 & $\mathrm{~N}$ \\
\hline & Kenya & 0.083 & 0.034 & -0.008 & -0.007 & -0.007 & 0.048 & 0.062 & 0.479 & $\mathrm{~N}$ & -0.155 & 0.339 & -1.432 & $\mathrm{~N}$ & 0.069 & 0.317 & 0.845 & $\mathrm{~N}$ \\
\hline & Mauritius & 0.302 & 0.030 & -0.004 & -0.003 & -0.003 & 0.001 & -0.163 & 0.043 & $\mathrm{~N}$ & -0.102 & -0.057 & -0.949 & $\mathrm{~N}$ & 0.373 & 0.060 & $4.502 * * *$ & $\mathrm{Y}$ \\
\hline & Morocco & 0.059 & 0.024 & 0.028 & 0.028 & 0.026 & 0.026 & -0.250 & -0.016 & $\mathrm{~N}$ & -0.126 & -0.250 & -1.489 & $\mathrm{~N}$ & 0.055 & -0.160 & 0.320 & $\mathrm{~N}$ \\
\hline & Namibia & 0.376 & 0.037 & 0.366 & 0.329 & 0.323 & 0.499 & 0.362 & 1.152 & $\mathrm{~N}$ & 0.084 & 0.745 & $-2.419^{* *}$ & $\mathrm{~N}$ & 0.261 & 0.809 & -0.694 & $\mathrm{~N}$ \\
\hline & Nigeria & 0.027 & 0.038 & 0.105 & 0.106 & 0.086 & -0.492 & -0.171 & $-6.40 * * *$ & $\mathrm{~N}$ & -0.448 & -0.195 & $-6.38 * * *$ & $\mathrm{~N}$ & -0.054 & 0.225 & -1.573 & $\mathrm{~N}$ \\
\hline & South A & 0.435 & 0.030 & 0.358 & 0.321 & 0.302 & 0.648 & 0.151 & $2.604 * *$ & $\mathrm{Y}$ & 0.198 & 0.471 & -1.188 & $\mathrm{~N}$ & 0.342 & 0.688 & 0.446 & $\mathrm{~N}$ \\
\hline & Tunisia & 0.258 & 0.016 & 0.166 & 0.117 & 0.117 & 0.233 & -0.399 & 0.582 & $\mathrm{~N}$ & 0.150 & 0.221 & 0.312 & $\mathrm{~N}$ & 0.311 & 0.228 & $2.198 * *$ & Y \\
\hline \multirow{10}{*}{$\begin{array}{l}\text { Middle } \\
\text { East }\end{array}$} & Dhabi & -0.069 & 0.030 & -0.051 & -0.050 & -0.041 & 0.235 & 0.107 & 2.566 ** & $\mathrm{Y}$ & -0.124 & 0.145 & -0.713 & $\mathrm{~N}$ & -0.066 & 0.686 & -0.275 & $\mathrm{~N}$ \\
\hline & Bahrain & 0.017 & 0.015 & -0.032 & -0.033 & -0.028 & 0.483 & -0.033 & $5.160 * * *$ & $\mathrm{Y}$ & 0.181 & -0.089 & $2.095 * *$ & $\mathrm{Y}$ & -0.004 & 0.235 & 0.268 & $\mathrm{~N}$ \\
\hline & Dubai & -0.085 & 0.039 & -0.027 & -0.166 & -0.021 & -0.152 & 0.110 & -1.089 & $\mathrm{~N}$ & -0.166 & 0.094 & -0.002 & $\mathrm{~N}$ & -0.096 & 0.727 & -0.830 & $\mathrm{~N}$ \\
\hline & Israel & 0.264 & 0.028 & 0.569 & 0.522 & 0.477 & 0.731 & -0.180 & 1.414 & $\mathrm{~N}$ & 0.281 & 0.052 & $-2.380^{* * *}$ & $\mathrm{~N}$ & 0.077 & 0.338 & -4.829 & Y \\
\hline & Jordan & 0.015 & 0.031 & 0.050 & 0.045 & 0.032 & 0.166 & -0.204 & 0.998 & $\mathrm{~N}$ & 0.034 & -0.017 & -0.106 & $\mathrm{~N}$ & 0.007 & 1.009 & -0.269 & $\mathrm{~N}$ \\
\hline & Kuwait & -0.085 & 0.026 & n.a & -0.007 & n.a & n.a & n.a & n.a & & n.a & n.a & n.a & & n.a & n.a & n.a & \\
\hline & Lebanon & 0.200 & 0.033 & 0.233 & 0.239 & 0.178 & 0.148 & -0.051 & -0.727 & $\mathrm{~N}$ & 0.191 & -0.106 & -0.463 & $\mathrm{~N}$ & 0.167 & 0.653 & -0.124 & $\mathrm{~N}$ \\
\hline & Oman & -0.217 & 0.031 & 0.104 & 0.087 & 0.072 & 0.012 & 0.181 & -0.796 & $\mathrm{~N}$ & -0.204 & 0.680 & $-2.92 * * *$ & $\mathrm{~N}$ & -0.201 & 1.453 & $-3.14 * * *$ & $\mathrm{~N}$ \\
\hline & Qatar & -0.133 & 0.040 & -0.035 & -0.030 & -0.026 & 0.198 & -0.123 & $2.063^{* *}$ & $\mathrm{Y}$ & -0.092 & 0.196 & -0.598 & $\mathrm{~N}$ & -0.142 & 0.540 & -1.289 & $\mathrm{~N}$ \\
\hline & Saudi A & 0.012 & 0.047 & 0.074 & 0.052 & 0.053 & -0.366 & -0.351 & $-4.21 * * *$ & $\mathrm{~N}$ & -0.099 & 0.294 & -1.474 & $\mathrm{~N}$ & -0.002 & 0.267 & -0.606 & $\mathrm{~N}$ \\
\hline \multirow{11}{*}{ Asia } & India & 0.264 & 0.038 & 0.223 & 0.266 & 0.256 & 0.272 & 0.559 & 0.426 & $\mathrm{~N}$ & 0.200 & 0.637 & -0.639 & $\mathrm{~N}$ & 0.161 & 0.773 & -1.067 & $\mathrm{~N}$ \\
\hline & Indonesia & 0.057 & 0.040 & 0.107 & 0.165 & 0.169 & 0.490 & 1.441 & $3.566 * * *$ & $\mathrm{Y}$ & 0.267 & 1.392 & 0.983 & $\mathrm{~N}$ & -0.021 & 1.287 & $-2.142 * *$ & $\mathrm{~N}$ \\
\hline & Malaysia & 0.100 & 0.026 & 0.152 & 0.195 & 0.208 & 0.679 & 0.780 & 5.338*** & $\mathrm{Y}$ & 0.352 & 0.872 & 1.521 & $\mathrm{~N}$ & 0.012 & 0.632 & $2.222 * *$ & Y \\
\hline & Mongolia & 0.062 & 0.046 & -0.203 & -0.253 & -0.270 & -0.140 & 0.258 & 0.543 & $\mathrm{~N}$ & -0.094 & -0.009 & 1.544 & $\mathrm{~N}$ & 0.053 & -0.138 & $3.782 * * * *$ & Y \\
\hline & Pakistan & 0.021 & 0.037 & 0.047 & 0.052 & 0.043 & 0.318 & 0.072 & $2.420 * *$ & $\mathrm{Y}$ & 0.342 & -0.077 & $2.906 * * *$ & $\mathrm{Y}$ & -0.026 & 0.382 & -0.764 & $\mathrm{~N}$ \\
\hline & Philippines & 0.361 & 0.040 & 0.122 & 0.176 & 0.171 & 0.701 & 0.817 & $6.113 * * *$ & $\mathrm{Y}$ & 0.537 & 0.545 & $3.712 * * *$ & Y & 0.299 & 0.650 & 1.432 & $\mathrm{~N}$ \\
\hline & S. Korea & 0.469 & 0.034 & 0.020 & 0.035 & 0.034 & 0.527 & 1.724 & $5.060 * * *$ & $\mathrm{Y}$ & 0.477 & 1.348 & 4.734*** & Y & 0.350 & 1.410 & $3.687 * * *$ & $\mathrm{Y}$ \\
\hline & Sri Lanka & 0.204 & 0.027 & -0.006 & -0.005 & -0.004 & -0.127 & -0.216 & -1.056 & $\mathrm{~N}$ & 0.434 & -0.271 & $4.687 * * *$ & $\mathrm{Y}$ & 0.286 & 0.017 & $3.362 * * *$ & Y \\
\hline & Taiwan & 0.429 & 0.035 & -0.034 & -0.050 & -0.048 & 0.639 & 1.028 & $7.839 * * *$ & $\mathrm{Y}$ & 0.311 & 0.945 & 3.711*** & $\mathrm{Y}$ & 0.355 & 1.105 & $4.876 * * *$ & Y \\
\hline & Thailand & 0.355 & 0.037 & 0.109 & 0.121 & 0.111 & 0.605 & 0.374 & $4.908 * * *$ & $\mathrm{Y}$ & 0.353 & 0.527 & $2.282 * *$ & $\mathrm{Y}$ & 0.296 & 0.815 & $2.087 * *$ & Y \\
\hline & Vietnam & 0.204 & 0.060 & 0.172 & 0.109 & 0.098 & 0.687 & -0.327 & $\mathbf{5 . 1 6 9} * * *$ & $\mathrm{Y}$ & 0.299 & 0.155 & $1.862^{*}$ & $\mathrm{Y}$ & 0.165 & 0.416 & 0.744 & $\mathrm{~N}$ \\
\hline \multirow{9}{*}{$\begin{array}{l}\text { Latin } \\
\text { America }\end{array}$} & 7 & 0.5 & 0.0 & 0.538 & & & & 0.746 & & $\mathrm{~N}$ & 0.565 & 0.407 & & $\mathrm{~N}$ & 0.2 & 0.976 & & $\mathrm{~N}$ \\
\hline & Brazil & 0.773 & 0.043 & 0.724 & 0.728 & 0.601 & 0.765 & 0.586 & 0.352 & $\mathrm{~N}$ & 0.640 & 0.550 & -0.843 & $\mathrm{~N}$ & 0.557 & 0.900 & -0.482 & $\mathrm{~N}$ \\
\hline & Chile & 0.690 & 0.03 & 0.453 & 0.454 & 0.326 & 0.589 & 1.044 & 1.174 & $\mathrm{~N}$ & 0.577 & 1.035 & 1.189 & $\mathrm{~N}$ & 0.434 & 1.228 & 1.198 & $\mathrm{~N}$ \\
\hline & Columbia & 0.475 & 0.032 & 0.316 & 0.300 & 0.252 & 0.359 & 0.142 & 0.370 & $\mathrm{~N}$ & 0.567 & 0.289 & $2.665^{* * *}$ & $\mathrm{Y}$ & 0.394 & 0.377 & 1.591 & $\mathrm{~N}$ \\
\hline & Costa Rica & -0.020 & 0.028 & -0.108 & -0.097 & -0.123 & -0.111 & -0.376 & -0.031 & $\mathrm{~N}$ & -0.231 & -0.235 & -1.294 & $\mathrm{~N}$ & -0.120 & -0.309 & 0.033 & $\mathrm{~N}$ \\
\hline & Ecuador & 0.030 & 0.029 & 0.145 & 0.047 & 0.034 & 0.017 & -0.659 & -1.106 & $\mathrm{~N}$ & 0.022 & 2.360 & -0.236 & $\mathrm{~N}$ & 0.006 & 1.517 & -0.308 & $\mathrm{~N}$ \\
\hline & Mexico & 0.774 & 0.037 & 0.657 & 0.655 & 0.537 & 0.761 & 0.430 & 0.898 & $\mathrm{~N}$ & 0.820 & 0.442 & 1.607 & $\mathrm{~N}$ & 0.614 & 0.715 & 0.857 & $\mathrm{~N}$ \\
\hline & Peru & 0.422 & 0.052 & -0.045 & -0.046 & -0.029 & 0.824 & 1.184 & $15.092 * * * *$ & Y & 0.555 & 1.072 & $7.210 * * *$ & $\mathrm{Y}$ & 0.242 & 1.268 & $3.117 * * *$ & Y \\
\hline & Venezuela & 0.119 & 0.034 & 0.042 & 0.037 & 0.044 & 0.229 & -0.301 & 1.642 & $\mathrm{~N}$ & 0.285 & -0.114 & $2.452 * *$ & $\mathrm{Y}$ & 0.201 & -0.216 & $1.760 *$ & Y \\
\hline
\end{tabular}

The table shows the unconditional (adjusted) cross market correlation coefficients ( $\rho$ ) and standard deviations for the US and other stock markets. Test statistics is obtained from t-transformations. The stable period is defined as the 14-month pre-crisis period (June 08, 2006 to August 09, 2007). The short-term turmoil period is defined as the four-month crisis period (August 09, 2007 to December 06, 2007). The medium-term period is the stable period plus the long-term turmoil period (June 08, 2006 to November 13,2008). Contagion (Co) occurs (Y) when the test statistics is greater than the critical values. No contagion (N) occurs when the test statistics is less than or equal to the critical value $* * * * * *$. represent significance at 10\%, 5\% and $1 \%$ respectively. (nt+ns-4) degrees of freedom for the t-statistics are (66+61-4); (35+61-4); $(17+61-4)$ for the long, medium and short terms respectively. $\sigma$ : represents the standard deviation. $\rho^{*} s t p, \rho * m t p, \rho * 1 t p$ denote adjusted correlation coefficients for the short, medium and long term periods respectively. $\delta$ : correlation coefficient adjuster. 


\subsection{Presentation of results and discussion}

As shown in Tables 1-2, contagion results based on significant shifts in conditional (unadjusted) correlation coefficients are robust to adjusted (unconditional) correlations. Broadly, the following effects of the financial crisis could be observed: (1) with the exceptions of India and Dhaka, Asian markets were worst hit; (2) but for Peru, Venezuela and Columbia, Latin American countries were least affected and; (3) Africa and Middle East emerging markets were averagely contaminated with the exceptions of Kenya, Namibia, Nigeria, Morocco, Dubai, Jordan, Israel, Oman, Saudi Arabia and Lebanon.

The quasi-immunity of Latin American countries to this recent global financial meltdown was not unexpected. Given its relative important history in the management of financial crises, the continent was the most prepared. Current trends show that, Latin America has improved since the Russian crisis, which has given countries in the continent some leeway (particularly in monetary policy) to implement measures that attenuate the effect of crises. Latin America and the Caribbean countries have built-up to 400 billion dollars in international reserves and have substantially reduced their dollar-denominated debt (especially within the banking system). For example, lower levels of debt dollarization has allowed Brazil to loosen monetary policy in the face of the credit crunch in ways that many countries could not in the post Russian crisis era. In the wake of the financial crisis, Latin American countries swiftly depreciated their currencies without getting into the turmoil. From a fiscal standpoint, many of these countries have saved a considerable amount of their tax income on extra revenue from commodity bonanza at the turn of the century. For instance, Chile has spent only $34 \%$ and kept the rest of increased tax collected in a special fund. Hence, even if the crisis had affected these countries, they would still have had the leeway of increasing spending while lowering taxes, in order to easily recover from recession.

Results from Africa were not entirely unexpected. With the exceptions of Kenya, Namibia, Nigeria and Morocco, African stock markets are contaminated in at least one time horizon. This reflects the increasing connection between African markets with global capital flows. Accordingly, African markets are growing in size, liquidity and degree of foreign participation. However misleading it may be to equate contagion to integration, a logical extension of the results could make a case for African equity markets global integration.

Turning to the Middle East, but for Israel, Oman and Saudi Arabia, oil exporting countries (Bahrain and Qatar) have been contaminated while, with the exception of Abu Dhabi, non-oil producing states (Dubai, Jordan, Lebanon) have remained unaffected. Consistent with Anoruo and Mustafa (2007) (on the relation between oil and stock prices, 
where causality runs from the Dow Jones Industrial Average (DJIA) to oil prices and not vice versa), the DJIA which is our base criterion in this study negatively affected oil prices (which in-turn has had a toll on the stock markets of oil exporting countries).

Whereas Dhaka and India in Asia have remained uncontaminated, China and Mongolia have been affected only in the short- and long-run horizons respectively. Other emerging markets have also been affected at least in two time-horizons. The unexpected speed and force with which the global financial crisis has affected Asian economies could be explained from trade channels. The region currently has deep economic integration with the rest of the world, especially developments in the United States. A case in point is the loss in export volume growth in Western Asia from 6.4\% in 2006 to $-0.6 \%$ in 2007. Conversely, the fact that India has been broadly unaffected is not unexpected. India has a completely different approach to financial globalization. Whereas the Indian current account was fully opened on a gradual basis in the 1990s, a more calibrated approach has been followed to the opening of the capital account and subsequently the financial sector. This approach is consistent with the available weight of empirical evidence on the benefits of capital account liberalization for acceleration of economic growth, particularly in emerging economies. Further evidence suggests that, the greatest gains are obtained from openness to foreign direct investment followed by portfolio investment. Benefits resulting from external debt flows are questionable until greater domestic financial market development has taken place (Henry, 2007; Asongu, 2012c). As a policy implication, policy making bodies should reconsider Latin American monetary and fiscal strategies in the fight against external financial shocks. Also, the validity of India's financial liberalization strategy could serve as a guide for governments in other developing countries ${ }^{8}$.

\section{The 2011 Japanese earthquake, tsunami and nuclear crises}

Natural disasters may inflict significant damage upon international financial markets. The purpose of this section is to investigate if any contagion effect occurred in the immediate aftermath of the Japanese earthquake, tsunami and subsequent nuclear crises.

\footnotetext{
"Whereas the Indian current account has been opened fully though gradually in the 1990s, a more calibrated approach has been followed in the opening of the capital account and subsequently the financial sector. This approach is consistent with the weight of available empirical evidence on the benefits of capital account liberalization for acceleration of economic growth, particularly in emerging economies. Evidence suggests that the greatest gains are obtained from openness to foreign direct investment followed by portfolio investment. Benefits resulting from external debt flows are questionable until greater domestic financial market development has taken place" (Henry, 2007).
} 


\subsection{Data}

To investigate the correlations among returns of the Japanese daily stock index (exchange rate) and 33 international stock indices (exchange rates) returns, the Japanese equity and foreign exchange markets are the base criteria. Thus, we examine whether comovements among national stock and foreign exchange markets increased significantly after the major earthquake, tsunami and nuclear disasters. The sample period is divided into two sections: the 12-month pre-earthquake period (March 11, 2010 to March 10, 2011) and the 2month post-earthquake period (March 11, 2011 to May 10, 2011) ${ }^{9}$. Whereas the stability period is defined as the pre-earthquake period, the turbulent (turmoil) period is the postearthquake period. In order to ensure robustness of our findings, the turmoil period is further divided into two equal sections: the 1-month (short-term) post-earthquake period (March 11, 2011 to April 10, 2011) and, the 2-month (medium-term) post-earthquake (March 11, 2011 to May 10, 2011). The number of days is respectively 365, 31, 62 days for the stable, short-term turmoil and medium-term turmoil periods. Data used in the study is obtained from Bloomberg's database. In the computing stock returns, last values are carried over for nontrading days. The US dollar is used as the common " $x$ " unit of foreign currency for each unit of national/regional currency in the computation of exchange rate returns. The use of local currency index return is consistent with Forbes and Rigobon who have shown that, using dollar or local indices will produce similar results.

\subsection{Methodology}

Borrowing from Forbes and Rigobon, contagion is a significant increase in market comovements after a shock has occurred in one country.

The coefficient of correlation is defined as:

$$
\rho=\frac{\sigma_{x y}}{\sigma_{x} \sigma_{y}}
$$

Where: ' $x$ ' represents the base criterion and ' $y$ ' an international market. This correlation coefficient is adjusted in the following manner to take account of heteroscedasticity:

$$
\rho^{*}=\frac{\rho}{\left.\sqrt{1+\delta\left[1-(\rho)^{2}\right.}\right]}
$$

Where:

\footnotetext{
${ }^{9}$ Differences in pre-earthquake and post-earthquake sample periods are in line with Collins and Biekpe (2003); Lee et al. (2007) and Asongu (2011, 2012a).
} 
$\delta=\frac{\sigma_{x x}^{h}}{\sigma_{x x}^{l}}-1$

It measures the change in high-period volatility against volatility in the low-period. Whereas the crisis interval is used as the high volatility period, the tranquil (or stable-period) represents the low volatility period. Contagion is accordingly measured as the significant increase of adjusted correlation coefficients in time-varying turmoil periods against the stability period.

Consistent with Lee et al. (2007), the following hypotheses are tested:

$H_{o}: \rho_{t}-\rho_{s} \leq 0$ versus $H_{1}: \rho_{t}-\rho_{s}>0$

Where, $\rho \mathrm{t}$ is the adjusted correlation coefficient during the turmoil period and $\rho \mathrm{s}$ the adjusted correlation coefficient for the stable period. A comparison of the variation in correlation between the stable and crisis periods is then analyzed. The null hypothesis $(H O)$ is the hypothesis of no contagion whereas $H 1$ is the alternative hypothesis for the presence of contagion. Fisher's $\mathrm{Z}$ transformations of correlation coefficients are employed to test pairwise cross-country significance. The Fisher's Z-transformations change standard coefficients to normally distributed $\mathrm{Z}$ variables. Therefore, prior to hypothesis testing, $\rho$ values must be converted to $\mathrm{Zr}$ values.

$$
\begin{aligned}
& H_{o}: \rho_{t}-\rho_{s} \leq 0 \Rightarrow H_{o}: Z_{r t}-Z_{r s} \leq 0 \\
& H_{1}: \rho_{t}-\rho_{s}>0 \Rightarrow H_{1}: Z_{r t}-Z_{r s}>0
\end{aligned}
$$

Where:

$$
\begin{aligned}
& Z_{r t}=\frac{1}{2} \ln \left(\frac{1+\rho_{t}}{1-\rho_{t}}\right) \\
& Z_{r s}=\frac{1}{2} \ln \left(\frac{1+\rho_{s}}{1-\rho_{s}}\right) \\
& Z=\frac{Z_{r t}-Z_{r s}}{\sqrt{\left(1 /\left(n_{t}-3\right)\right)+\left(1 /\left(n_{s}-3\right)\right)}}
\end{aligned}
$$

\subsection{Empirical results and discussion}

\subsubsection{Contagion effect in international stock indexes returns after earthquake}

Table 3 below shows the conditional (unadjusted) correlation coefficients of international stock indices for the 2011 Japanese Tsunami. Cross-market correlations of stock index returns are analyzed before and after the earthquake of March 11, 2011. But for China, Taiwan, New Zealand, Argentina, Bahrain, Egypt, Saudi Arabia and South Africa; cross- 
market correlations between Japan and most countries in the sample during stable period are higher than those during medium-term turmoil period. For the short-run interval, correlations are strengthened for China, Hong Kong, Taiwan, New Zealand, Argentina, Germany, South Korea, Australia, Bahrain, South Africa and Saudi Arabia. There is significant evidence of contagion in Saudi Arabia, Taiwan, Bahrain and South Africa for the short-term turmoil period and only in Bahrain and Saudi Arabia for the medium-term turmoil interval. Relatively for the most part, volatilities of most countries during the stable period are higher than those during turmoil periods (short and medium terms). Table 4 presents unconditional correlation coefficients. These adjusted correlations are higher than their unadjusted counterparts in Table 3. Ultimately, the findings in Table 3 are robust to those in Table 4.

\subsubsection{Contagion effect in international exchange rates returns after earthquake}

Table 5 presents findings for exchange rate conditional (unadjusted) correlation coefficients. Accordingly, cross-market correlations during turmoil periods are higher than those during the stable period. Strengthened cross-market correlations with insignificant evidence of contagion are noticeable for the Argentine Peso (ARS), Thai Baht (THB), Egyptian Pound (EGP), and Qatari Riyal (QAR) for the short-term turmoil period. Looking at the medium-term, the Chinese Yuan (RMB), Canadian Dollar (CAD), Egyptian pound (EGP), Qatari Riyal (QAR) and Emirati dirham (AED) have also witnessed insignificant stronger comovements with the Japanese Yen (JPY). Adjusted findings in Table 6 confirm those in Table 5. Ultimately, no national/regional exchange market is found to have suffered from contagion two months in the aftermath of the Japanese earthquake and the ensuing collateral disasters. 
Table 3: International stock indices returns conditional (unadjusted) correlation coefficients in 2011 Japanese earthquake

\begin{tabular}{|c|c|c|c|c|c|c|c|c|c|c|c|c|c|}
\hline \multirow[b]{2}{*}{ Regions } & \multirow[b]{2}{*}{ Countries } & \multicolumn{2}{|c|}{ Full period } & \multicolumn{2}{|c|}{ Stable Period } & \multicolumn{4}{|c|}{ Short-term turmoil period } & \multicolumn{4}{|c|}{ Medium-term turmoil period } \\
\hline & & $\rho$ & $\sigma$ & $\rho$ & $\sigma$ & $\rho$ & $\sigma$ & Z-test & $\mathrm{Co}$ & $\rho$ & $\sigma$ & Z-test & $\mathrm{Co}$ \\
\hline \multirow{5}{*}{$\begin{array}{l}\text { South Asia } \\
\text { and South- } \\
\text { East Asia }\end{array}$} & India & 0.288 & 0.009 & 0.343 & 0.009 & 0.247 & 0.009 & -0.538 & $\mathrm{~N}$ & 0.171 & 0.009 & -1.321 & $\mathrm{~N}$ \\
\hline & Malaysia & 0.372 & 0.005 & 0.405 & 0.005 & 0.392 & 0.005 & -0.080 & $\mathrm{~N}$ & 0.348 & 0.005 & -0.474 & $\mathrm{~N}$ \\
\hline & Philippines & 0.317 & 0.009 & 0.357 & 0.009 & 0.295 & 0.009 & -0.353 & $\mathrm{~N}$ & 0.266 & 0.008 & -0.715 & $\mathrm{~N}$ \\
\hline & Singapore & n.a & 0.000 & n.a & 0.000 & n.a & 0.000 & n.a & n.a & n.a & 0.000 & & n.a \\
\hline & Thailand & 0.308 & 0.009 & 0.361 & 0.010 & 0.278 & 0.008 & -0.470 & $\mathrm{~N}$ & 0.209 & 0.008 & -1.180 & $\mathrm{~N}$ \\
\hline \multirow{4}{*}{$\begin{array}{l}\text { East Asia } \\
\text { and North- } \\
\text { East Asia }\end{array}$} & China & 0.283 & 0.011 & 0.309 & 0.012 & 0.477 & 0.007 & 1.022 & $\mathrm{~N}$ & 0.321 & 0.007 & 0.100 & $\mathrm{~N}$ \\
\hline & Hong Kong & 0.510 & 0.009 & 0.542 & 0.009 & 0.574 & 0.009 & 0.240 & $\mathrm{~N}$ & 0.525 & 0.008 & -0.166 & $\mathrm{~N}$ \\
\hline & Taiwan & 0.587 & 0.008 & 0.591 & 0.008 & 0.781 & 0.008 & $1.881 *$ & $\mathrm{Y}$ & 0.694 & 0.008 & 1.247 & $\mathrm{~N}$ \\
\hline & South Korea & 0.575 & 0.008 & 0.606 & 0.008 & 0.660 & 0.008 & 0.458 & $\mathrm{~N}$ & 0.566 & 0.008 & -0.437 & $\mathrm{~N}$ \\
\hline \multirow{2}{*}{ Australasia } & Australia & -0.004 & 0.008 & 0.000 & 0.008 & 0.073 & 0.007 & 0.373 & $\mathrm{~N}$ & -0.021 & 0.007 & -0.147 & $\mathrm{~N}$ \\
\hline & New Zealand & 0.440 & 0.004 & 0.459 & 0.004 & 0.609 & 0.004 & 1.080 & $\mathrm{~N}$ & 0.515 & 0.004 & 0.525 & $\mathrm{~N}$ \\
\hline \multirow{3}{*}{$\begin{array}{l}\text { North } \\
\text { America }\end{array}$} & Canada & -0.055 & 0.197 & -0.013 & 0.208 & -0.441 & 0.125 & -2.348 & $\mathrm{~N}$ & -0.343 & 0.110 & -2.455 & $\mathrm{~N}$ \\
\hline & U.S.A & 0.176 & 0.012 & 0.217 & 0.013 & 0.054 & 0.008 & -0.848 & $\mathrm{~N}$ & 0.074 & 0.007 & -1.041 & $\mathrm{~N}$ \\
\hline & Mexico & 0.159 & 0.007 & 0.208 & 0.007 & 0.048 & 0.006 & -0.831 & $\mathrm{~N}$ & 0.027 & 0.006 & -1.310 & $\mathrm{~N}$ \\
\hline \multirow{3}{*}{$\begin{array}{l}\text { South } \\
\text { America }\end{array}$} & Argentina & 0.174 & 0.012 & 0.163 & 0.013 & 0.312 & 0.011 & 0.807 & $\mathrm{~N}$ & 0.269 & 0.010 & 0.795 & $\mathrm{~N}$ \\
\hline & Brazil & 0.076 & 0.010 & 0.120 & 0.010 & -0.033 & 0.006 & -0.783 & $\mathrm{~N}$ & -0.069 & 0.008 & -1.351 & $\mathrm{~N}$ \\
\hline & Chile & 0.117 & 0.007 & 0.178 & 0.007 & -0.086 & 0.008 & -1.357 & $\mathrm{~N}$ & -0.035 & 0.007 & -1.532 & $\mathrm{~N}$ \\
\hline \multirow{7}{*}{ Europe } & France & 0.321 & 0.012 & 0.366 & 0.012 & 0.253 & 0.011 & -0.639 & $\mathrm{~N}$ & 0.254 & 0.010 & -0.883 & $\mathrm{~N}$ \\
\hline & Poland & 0.218 & 0.008 & 0.287 & 0.008 & -0.045 & 0.006 & -1.735 & $\mathrm{~N}$ & 0.013 & 0.006 & -2.014 & $\mathrm{~N}$ \\
\hline & Germany & 0.325 & 0.009 & 0.366 & 0.009 & 0.350 & 0.012 & 0.083 & $\mathrm{~N}$ & 0.334 & 0.011 & -0.012 & $\mathrm{~N}$ \\
\hline & Italy & 0.248 & 0.013 & 0.292 & 0.013 & 0.142 & 0.009 & -0.806 & $\mathrm{~N}$ & 0.169 & 0.009 & -0.928 & $\mathrm{~N}$ \\
\hline & Holland & 0.332 & 0.010 & 0.378 & 0.010 & 0.296 & 0.008 & -0.473 & $\mathrm{~N}$ & 0.271 & 0.008 & -0.851 & $\mathrm{~N}$ \\
\hline & Spain & 0.193 & 0.015 & 0.255 & 0.016 & -0.116 & 0.009 & -1.923 & $\mathrm{~N}$ & -0.001 & 0.009 & -1.860 & $\mathrm{~N}$ \\
\hline & U.K & 0.292 & 0.009 & 0.361 & 0.009 & 0.135 & 0.008 & -1.234 & $\mathrm{~N}$ & 0.129 & 0.008 & -1.764 & $\mathrm{~N}$ \\
\hline \multirow{8}{*}{$\begin{array}{l}\text { Middle } \\
\text { East and } \\
\text { Africa }\end{array}$} & Bahrain & 0.006 & 0.005 & -0.050 & 0.005 & 0.290 & 0.006 & $1.774 *$ & $\mathrm{Y}$ & 0.207 & 0.005 & $1.850 *$ & $\mathrm{Y}$ \\
\hline & Egypt & 0.116 & 0.013 & 0.104 & 0.011 & 0.098 & 0.028 & -0.027 & $\mathrm{~N}$ & 0.131 & 0.022 & 0.198 & $\mathrm{~N}$ \\
\hline & Jordan & -0.035 & 0.005 & -0.020 & 0.005 & -0.101 & 0.006 & -0.413 & $\mathrm{~N}$ & -0.097 & 0.005 & -0.554 & $\mathrm{~N}$ \\
\hline & Kuwait & -0.073 & 0.005 & -0.026 & 0.005 & -0.298 & 0.006 & -1.431 & $\mathrm{~N}$ & -0.256 & 0.004 & -1.679 & $\mathrm{~N}$ \\
\hline & Qatar & 0.019 & 0.009 & 0.046 & 0.009 & -0.080 & 0.009 & -0.641 & $\mathrm{~N}$ & -0.064 & 0.008 & -0.785 & $\mathrm{~N}$ \\
\hline & Saudi Arabia & 0.182 & 0.011 & 0.117 & 0.011 & 0.493 & 0.013 & $2.154 * *$ & $\mathrm{Y}$ & 0.457 & 0.010 & $2.678 * * *$ & $\mathrm{Y}$ \\
\hline & UAE & 0.080 & 0.006 & 0.109 & 0.006 & -0.055 & 0.006 & -0.836 & $\mathrm{~N}$ & 0.010 & 0.005 & -0.706 & $\mathrm{~N}$ \\
\hline & South Africa & 0.348 & 0.009 & 0.343 & 0.009 & 0.634 & 0.008 & $1.994 * *$ & $\mathrm{Y}$ & 0.434 & 0.009 & 0.766 & $\mathrm{~N}$ \\
\hline Other & Russia & 0.290 & 0.012 & 0.378 & 0.012 & 0.007 & 0.010 & -1.992 & $\mathrm{~N}$ & 0.069 & 0.012 & -2.338 & $\mathrm{~N}$ \\
\hline
\end{tabular}

Note $1: *, * * * * *$ statistical significance at $10 \%, 5 \%$ and $1 \%$ respectively.

Note 2: The table shows conditional (unadjusted) cross-market correlation coefficients $(\rho)$ and standard deviations $(\sigma)$ for Japan and other stock indexes. The test statistics are obtained from Fisher Z transformations. The stable period is defined as the 12-month pre-earthquake period (March 11, 2010 to March 10, 2011). The short-term turmoil period is defined as the 1-month post-earthquake period (March 11, 2011 to April 10, 2011). The medium-term turmoil period is defined as the 2-month post-earthquake period (March 11, 2011 to May 10, 2011). The full period is the stable period plus the medium-term turmoil period. Co: contagion .While "Y" denotes that the test statistics is greater than the critical value and contagion occurred, "N" indicates that the test statistics was less or equal to the critical value and no contagion occurred.

Note 3: Correlation coefficients are unadjusted for heteroscedasticity. 
Table 4: International stock indices returns unconditional (adjusted) correlation coefficients in 2011 Japanese earthquake

\begin{tabular}{|c|c|c|c|c|c|c|c|c|c|c|c|c|c|}
\hline \multirow[b]{2}{*}{ Regions } & \multirow[b]{2}{*}{ Countries } & \multicolumn{2}{|c|}{ Full period } & \multicolumn{2}{|c|}{ Stable Period } & \multicolumn{4}{|c|}{ Short-term turmoil period } & \multicolumn{4}{|c|}{ Medium-term turmoil period } \\
\hline & & $\rho$ & $\sigma$ & $\rho^{*}$ stp & $\rho^{*} \mathrm{mtp}$ & $\rho^{*}$ & $\delta$ & Z-test & Co & $\rho^{*}$ & $\delta$ & Z-test & Co \\
\hline \multirow{5}{*}{$\begin{array}{l}\text { South Asia } \\
\text { and South- } \\
\text { East Asia }\end{array}$} & India & 0.288 & 0.009 & 0.430 & 0.445 & 0.315 & -0.017 & -0.679 & $\mathrm{~N}$ & 0.229 & 0.032 & -1.745 & $\mathrm{~N}$ \\
\hline & Malaysia & 0.372 & 0.005 & 0.500 & 0.516 & 0.485 & -0.077 & -0.099 & $\mathrm{~N}$ & 0.451 & -0.111 & -0.609 & $\mathrm{~N}$ \\
\hline & Philippines & 0.317 & 0.009 & 0.445 & 0.460 & 0.373 & 0.007 & -0.443 & $\mathrm{~N}$ & 0.351 & -0.143 & -0.933 & $\mathrm{~N}$ \\
\hline & Singapore & n.a & 0.000 & n.a & n.a & n.a & n.a & n.a & n.a & n.a & n.a & n.a & n.a \\
\hline & Thailand & 0.308 & 0.009 & 0.450 & 0.465 & 0.352 & -0.165 & -0.591 & $\mathrm{~N}$ & 0.279 & -0.155 & -1.549 & $\mathrm{~N}$ \\
\hline \multirow{4}{*}{$\begin{array}{l}\text { East Asia } \\
\text { and North- } \\
\text { East Asia }\end{array}$} & China & 0.283 & 0.011 & 0.389 & 0.403 & 0.577 & -0.433 & 1.262 & $\mathrm{~N}$ & 0.418 & -0.387 & 0.130 & $\mathrm{~N}$ \\
\hline & Hong Kong & 0.510 & 0.009 & 0.642 & 0.658 & 0.674 & 0.048 & 0.283 & $\mathrm{~N}$ & 0.642 & -0.106 & -0.203 & $\mathrm{~N}$ \\
\hline & Taiwan & 0.587 & 0.008 & 0.690 & 0.706 & 0.852 & 0.047 & $2.119 * *$ & $\mathrm{Y}$ & 0.794 & -0.043 & 1.458 & $\mathrm{~N}$ \\
\hline & South Korea & 0.575 & 0.008 & 0.704 & 0.719 & 0.753 & 0.016 & 0.528 & $\mathrm{~N}$ & 0.682 & 0.092 & -0.523 & $\mathrm{~N}$ \\
\hline \multirow{2}{*}{ Australasia } & Australia & -0.004 & 0.008 & 0.000 & 0.000 & 0.095 & -0.706 & 0.485 & $\mathrm{~N}$ & -0.028 & -0.120 & -0.199 & $\mathrm{~N}$ \\
\hline & New Zealand & 0.440 & 0.004 & 0.558 & 0.574 & 0.707 & -0.119 & 1.283 & $\mathrm{~N}$ & 0.632 & -0.172 & 0.651 & $\mathrm{~N}$ \\
\hline \multirow{3}{*}{$\begin{array}{l}\text { North } \\
\text { America }\end{array}$} & Canada & -0.055 & 0.197 & -0.017 & -0.018 & -0.539 & -0.401 & -2.985 & $\mathrm{~N}$ & -0.445 & -0.473 & -3.277 & $\mathrm{~N}$ \\
\hline & U.S.A & 0.176 & 0.012 & 0.278 & 0.289 & 0.070 & -0.410 & -1.096 & $\mathrm{~N}$ & 0.100 & -0.458 & -1.401 & $\mathrm{~N}$ \\
\hline & Mexico & 0.159 & 0.007 & 0.267 & 0.278 & 0.063 & -0.174 & -1.074 & $\mathrm{~N}$ & 0.037 & -0.118 & -1.767 & $\mathrm{~N}$ \\
\hline \multirow{3}{*}{$\begin{array}{l}\text { South } \\
\text { America }\end{array}$} & Argentina & 0.174 & 0.012 & 0.210 & 0.219 & 0.393 & -0.139 & 1.029 & $\mathrm{~N}$ & 0.355 & -0.198 & 1.059 & $\mathrm{~N}$ \\
\hline & Brazil & 0.076 & 0.010 & 0.155 & 0.162 & -0.043 & -0.381 & -1.018 & $\mathrm{~N}$ & -0.094 & -0.228 & -1.833 & $\mathrm{~N}$ \\
\hline & Chile & 0.117 & 0.007 & 0.229 & 0.238 & -0.112 & 0.215 & -1.761 & $\mathrm{~N}$ & -0.048 & 0.038 & -2.073 & $\mathrm{~N}$ \\
\hline \multirow{7}{*}{ Europe } & France & 0.321 & 0.012 & 0.456 & 0.471 & 0.322 & -0.124 & -0.805 & $\mathrm{~N}$ & 0.336 & -0.188 & -1.153 & $\mathrm{~N}$ \\
\hline & Poland & 0.218 & 0.008 & 0.364 & 0.377 & -0.058 & -0.295 & -2.240 & $\mathrm{~N}$ & 0.017 & -0.295 & -2.702 & $\mathrm{~N}$ \\
\hline & Germany & 0.325 & 0.009 & 0.421 & 0.436 & 0.437 & 0.255 & 0.104 & $\mathrm{~N}$ & 0.434 & 0.142 & -0.015 & $\mathrm{~N}$ \\
\hline & Italy & 0.248 & 0.013 & 0.369 & 0.383 & 0.183 & -0.314 & -1.032 & $\mathrm{~N}$ & 0.227 & -0.306 & -1.232 & $\mathrm{~N}$ \\
\hline & Holland & 0.332 & 0.010 & 0.469 & 0.485 & 0.374 & -0.209 & -0.593 & $\mathrm{~N}$ & 0.358 & -0.242 & -1.108 & $\mathrm{~N}$ \\
\hline & Spain & 0.193 & 0.015 & 0.324 & 0.337 & -0.151 & -0.406 & -2.489 & $\mathrm{~N}$ & -0.001 & -0.443 & -2.504 & $\mathrm{~N}$ \\
\hline & U.K & 0.292 & 0.009 & 0.450 & 0.465 & 0.175 & -0.152 & -1.571 & $\mathrm{~N}$ & 0.175 & -0.120 & -2.333 & $\mathrm{~N}$ \\
\hline \multirow{8}{*}{$\begin{array}{l}\text { Middle } \\
\text { East and } \\
\text { Africa }\end{array}$} & Bahrain & 0.006 & 0.005 & -0.065 & -0.067 & 0.367 & 0.022 & $2.290 * *$ & $\mathrm{Y}$ & 0.276 & -0.156 & 2.501** & $\mathrm{Y}$ \\
\hline & Egypt & 0.116 & 0.013 & 0.134 & 0.140 & 0.127 & 1.605 & -0.036 & $\mathrm{~N}$ & 0.177 & 0.991 & 0.268 & $\mathrm{~N}$ \\
\hline & Jordan & -0.035 & 0.005 & -0.026 & -0.027 & -0.131 & 0.077 & -0.537 & $\mathrm{~N}$ & -0.132 & -0.159 & -0.752 & $\mathrm{~N}$ \\
\hline & Kuwait & -0.073 & 0.005 & -0.034 & -0.036 & -0.376 & 0.088 & -1.841 & $\mathrm{~N}$ & -0.339 & -0.157 & -2.257 & $\mathrm{~N}$ \\
\hline & Qatar & 0.019 & 0.009 & 0.059 & 0.062 & -0.104 & 0.037 & -0.834 & $\mathrm{~N}$ & -0.087 & -0.133 & -1.066 & $\mathrm{~N}$ \\
\hline & Saudi Arabia & 0.182 & 0.011 & 0.152 & 0.158 & 0.594 & 0.170 & $2.705 * * *$ & $\mathrm{Y}$ & 0.572 & -0.111 & $3.502 * * *$ & $\mathrm{Y}$ \\
\hline & UAE & 0.080 & 0.006 & 0.141 & 0.147 & -0.071 & 0.012 & -1.087 & $\mathrm{~N}$ & 0.014 & -0.085 & -0.957 & $\mathrm{~N}$ \\
\hline & South Africa & 0.348 & 0.009 & 0.429 & 0.444 & 0.730 & -0.136 & $2.394 * *$ & $\mathrm{Y}$ & 0.548 & -0.036 & 0.980 & $\mathrm{~N}$ \\
\hline Other & Russia & 0.290 & 0.012 & 0.469 & 0.485 & 0.009 & -0.176 & -2.550 & $\mathrm{~N}$ & 0.094 & -0.006 & -3.100 & $\mathrm{~N}$ \\
\hline
\end{tabular}

Note $1: *, * * * *$ : statistical significance at $10 \%, 5 \%$ and $1 \%$ respectively.
Note 2: The table shows conditional (unadjusted) cross-market correlation coefficients $(\rho)$, adjuster for heteroscedasticity $(\delta)$ and standard deviations $(\sigma)$ for Japan and other stock indexes. The test statistics are obtained from Fisher Z transformations. The stable period is defined as the 12-month pre-earthquake period (March 11, 2010 to March 10, 2011). The short-term turmoil period is defined as the 1-month post-earthquake period from F 11,2011 the (March 11, 2011 to April 10, 2011). The medium-term turmoil period is defined as the 2-month post-earthquake period (March 11, 2011 to May 10, 2011). The full period is the stable period plus the medium-term turmoil period. Co: contagion. While "Y" denotes that the test statistics is greater than the critical value and contagion occurred, "N" indicates that the test statistics was less or equal to the critical value and no contagion occurred. $\rho^{*} \mathrm{stp}, \rho^{*} \mathrm{mtp}$, denote adjusted correlation coefficients for the short and medium term periods respectively. $\delta$ : correlation coefficient adjuster.

Note 3: Correlation coefficients are adjusted for heteroscedasticity using Equation 2. 
Table 5: International exchange rates returns conditional (unadjusted) correlation coefficients in 2011 Japanese earthquake

\begin{tabular}{|c|c|c|c|c|c|c|c|c|c|c|c|c|c|}
\hline \multirow[b]{2}{*}{ Regions } & \multirow[b]{2}{*}{ Countries } & \multicolumn{2}{|c|}{ Full period } & \multicolumn{2}{|c|}{ Stable Period } & \multicolumn{4}{|c|}{ Short-term turmoil period } & \multicolumn{4}{|c|}{ Medium-term turmoil period } \\
\hline & & $\rho$ & $\sigma$ & $\rho$ & $\sigma$ & $\rho$ & $\sigma$ & Z-test & Co & $\rho$ & $\sigma$ & Z-test & $\mathrm{Co}$ \\
\hline \multirow{5}{*}{$\begin{array}{l}\text { South Asia } \\
\text { and South- } \\
\text { East Asia }\end{array}$} & India & -0.136 & 0.004 & -0.130 & 0.005 & -0.282 & 0.002 & -0.807 & $\mathrm{~N}$ & -0.207 & 0.002 & -0.560 & $\mathrm{~N}$ \\
\hline & Malaysia & -0.197 & 0.004 & -0.191 & 0.005 & -0.332 & 0.002 & -0.773 & $\mathrm{~N}$ & -0.267 & 0.003 & -0.570 & $\mathrm{~N}$ \\
\hline & Philippines & -0.129 & 0.004 & -0.128 & 0.005 & -0.439 & 0.003 & -1.746 & $\mathrm{~N}$ & -0.143 & 0.003 & -0.105 & $\mathrm{~N}$ \\
\hline & Singapore & -0.029 & 0.003 & -0.015 & 0.004 & -0.197 & 0.003 & -0.940 & $\mathrm{~N}$ & -0.109 & 0.003 & -0.674 & $\mathrm{~N}$ \\
\hline & Thailand & 0.061 & 0.002 & 0.066 & 0.004 & 0.235 & 0.002 & 0.885 & $\mathrm{~N}$ & 0.023 & 0.002 & -0.307 & $\mathrm{~N}$ \\
\hline \multirow{4}{*}{$\begin{array}{l}\text { East Asia } \\
\text { and North- } \\
\text { East Asia }\end{array}$} & China & 0.030 & 0.001 & 0.018 & 0.004 & -0.090 & 0.001 & -0.551 & $\mathrm{~N}$ & 0.085 & 0.001 & 0.482 & $\mathrm{~N}$ \\
\hline & Hong Kong & -0.049 & 0.000 & -0.020 & 0.004 & -0.223 & 0.000 & -1.049 & $\mathrm{~N}$ & -0.225 & 0.000 & -1.481 & $\mathrm{~N}$ \\
\hline & Taiwan & -0.104 & 0.003 & -0.080 & 0.004 & -0.400 & 0.002 & -1.748 & $\mathrm{~N}$ & -0.251 & 0.002 & -1.256 & $\mathrm{~N}$ \\
\hline & South Korea & -0.242 & 0.007 & -0.226 & 0.007 & -0.500 & 0.005 & -1.630 & $\mathrm{~N}$ & -0.415 & 0.004 & -1.508 & $\mathrm{~N}$ \\
\hline \multirow[t]{2}{*}{ Australasia } & Australia & -0.080 & 0.007 & -0.042 & 0.007 & -0.428 & 0.006 & -2.120 & $\mathrm{~N}$ & -0.325 & 0.006 & -2.099 & $\mathrm{~N}$ \\
\hline & New Zealand & -0.031 & 0.007 & 0.035 & 0.007 & -0.612 & 0.007 & -3.812 & $\mathrm{~N}$ & -0.419 & 0.006 & -3.432 & $\mathrm{~N}$ \\
\hline North & Canada & -0.272 & 0.006 & -0.275 & 0.006 & -0.390 & 0.004 & -0.662 & $\mathrm{~N}$ & -0.274 & 0.004 & 0.010 & $\mathrm{~N}$ \\
\hline America & Mexico & -0.338 & 0.005 & -0.339 & 0.006 & -0.521 & 0.003 & -1.151 & $\mathrm{~N}$ & -0.385 & 0.004 & -0.378 & $\mathrm{~N}$ \\
\hline South & Argentina & -0.030 & 0.001 & -0.026 & 0.001 & -0.024 & 0.001 & 0.010 & $\mathrm{~N}$ & -0.059 & 0.001 & -0.237 & $\mathrm{~N}$ \\
\hline America & Chile & 0.012 & 0.005 & 0.046 & 0.006 & -0.281 & 0.004 & -1.707 & $\mathrm{~N}$ & -0.234 & 0.004 & -2.028 & $\mathrm{~N}$ \\
\hline \multirow[t]{4}{*}{ Europe } & Euro & 0.166 & 0.006 & 0.211 & 0.006 & -0.204 & 0.004 & -2.151 & $\mathrm{~N}$ & -0.079 & 0.006 & -2.094 & $\mathrm{~N}$ \\
\hline & U.K & 0.043 & 0.005 & 0.073 & 0.005 & -0.211 & 0.005 & -1.467 & $\mathrm{~N}$ & -0.147 & 0.004 & -1.576 & $\mathrm{~N}$ \\
\hline & Bahrain & -0.021 & 0.006 & -0.014 & 0.000 & -0.029 & 0.000 & -0.077 & $\mathrm{~N}$ & -0.043 & 0.000 & -0.208 & $\mathrm{~N}$ \\
\hline & Egypt & 0.037 & 0.001 & 0.022 & 0.001 & 0.133 & 0.001 & 0.566 & $\mathrm{~N}$ & 0.194 & 0.001 & 1.239 & $\mathrm{~N}$ \\
\hline Middle & Jordan & -0.026 & 0.001 & -0.024 & 0.001 & -0.131 & 0.000 & -0.548 & $\mathrm{~N}$ & -0.036 & 0.000 & -0.088 & $\mathrm{~N}$ \\
\hline East and & Kuwait & 0.247 & 0.001 & 0.258 & 0.001 & 0.182 & 0.001 & -0.408 & $\mathrm{~N}$ & 0.187 & 0.001 & -0.533 & $\mathrm{~N}$ \\
\hline \multirow[t]{4}{*}{ Africa } & Qatar & 0.037 & 0.000 & 0.029 & 0.000 & 0.211 & 0.000 & 0.994 & $\mathrm{~N}$ & 0.178 & 0.000 & 1.072 & $\mathrm{~N}$ \\
\hline & Saudi Arabia & -0.027 & 0.000 & 0.005 & 0.000 & -0.334 & 0.000 & -1.795 & $\mathrm{~N}$ & -0.194 & 0.000 & -1.431 & $\mathrm{~N}$ \\
\hline & UAE & -0.086 & 0.000 & -0.094 & 0.001 & -0.211 & 0.005 & n.a & n.a & -0.037 & 0.000 & 0.406 & $\mathrm{~N}$ \\
\hline & South Africa & -0.130 & 0.007 & -0.074 & 0.007 & -0.601 & 0.007 & -3.170 & $\mathrm{~N}$ & -0.448 & 0.007 & -2.906 & $\mathrm{~N}$ \\
\hline Other & Russia & -0.140 & 0.004 & -0.132 & 0.005 & -0.377 & 0.003 & -1.347 & $\mathrm{~N}$ & -0.211 & 0.004 & -0.580 & $\mathrm{~N}$ \\
\hline
\end{tabular}

Note $1: * * *, * * *$ statistical significance at $10 \%, 5 \%$ and $1 \%$ respectively. n.a: the presence of zero exchange rate return for all periods of the series.

Note 2: The table shows conditional (unadjusted) cross-market correlation coefficients $(\rho)$ and standard deviations $(\sigma)$ for Japan and other stock indexes. The test statistics are obtained from Fisher Z transformations. The stable period is defined as the 12-month pre-earthquake period (March 11, 2010 to March 10, 2011). The short-term turmoil period is defined as the 1-month post-earthquake period (March 11, 2011 to April 10, 2011). The medium-term turmoil period is defined as the 2-month post-earthquake period (March 11, 2011 to May 10, 2011). The full period is the stable period plus the medium-term turmoil period. Co: contagion While "Y" denotes that the test statistics is greater than the critical value and contagion occurred, "N" indicates that the test statistics was less or equal to the critical value and no contagion occurred.

Note 3: Correlation coefficients are unadjusted for heteroscedasticity. 
Table 6: International exchange rates returns unconditional (adjusted) correlation coefficients in 2011 Japanese earthquake

\begin{tabular}{|c|c|c|c|c|c|c|c|c|c|c|c|c|c|}
\hline \multirow[b]{2}{*}{ Regions } & \multirow[b]{2}{*}{ Countries } & \multicolumn{2}{|c|}{ Full period } & \multicolumn{2}{|c|}{ Stable Period } & \multicolumn{4}{|c|}{ Short-term turmoil period } & \multicolumn{4}{|c|}{ Medium-term turmoil period } \\
\hline & & $\rho$ & $\sigma$ & $\rho^{*} \operatorname{stp}$ & $\rho^{*} m t p$ & $\rho^{*}$ & $\delta$ & Z-test & $\mathrm{Co}$ & $\rho^{*}$ & $\delta$ & Z-test & $\mathrm{Co}$ \\
\hline \multirow{5}{*}{$\begin{array}{l}\text { South Asia } \\
\text { and South- } \\
\text { East Asia }\end{array}$} & India & -0.136 & 0.004 & -0.181 & -0.168 & -0.379 & -0.487 & -1.104 & $\mathrm{~N}$ & -0.265 & -0.408 & -0.721 & $\mathrm{~N}$ \\
\hline & Malaysia & -0.197 & 0.004 & -0.262 & -0.245 & -0.441 & -0.510 & -1.045 & $\mathrm{~N}$ & -0.339 & -0.356 & -0.728 & $\mathrm{~N}$ \\
\hline & Philippines & -0.129 & 0.004 & -0.178 & -0.166 & -0.564 & -0.267 & -2.341 & $\mathrm{~N}$ & -0.184 & -0.281 & -0.136 & $\mathrm{~N}$ \\
\hline & Singapore & -0.029 & 0.003 & -0.021 & -0.020 & -0.270 & -0.154 & -1.304 & $\mathrm{~N}$ & -0.141 & -0.088 & -0.874 & $\mathrm{~N}$ \\
\hline & Thailand & 0.061 & 0.002 & 0.092 & 0.086 & 0.320 & -0.282 & 1.222 & $\mathrm{~N}$ & 0.030 & -0.194 & -0.399 & $\mathrm{~N}$ \\
\hline \multirow{4}{*}{$\begin{array}{l}\text { East Asia } \\
\text { and North- } \\
\text { East Asia }\end{array}$} & China & 0.030 & 0.001 & 0.024 & 0.023 & -0.126 & -0.142 & -0.769 & $\mathrm{~N}$ & 0.110 & 0.135 & 0.626 & $\mathrm{~N}$ \\
\hline & Hong Kong & -0.049 & 0.000 & -0.029 & -0.027 & -0.304 & 0.072 & -1.453 & $\mathrm{~N}$ & -0.287 & -0.099 & -1.913 & $\mathrm{~N}$ \\
\hline & Taiwan & -0.104 & 0.003 & -0.112 & -0.104 & -0.520 & -0.065 & -2.367 & $\mathrm{~N}$ & -0.320 & -0.085 & -1.617 & $\mathrm{~N}$ \\
\hline & South Korea & -0.242 & 0.007 & -0.308 & -0.289 & -0.628 & -0.322 & -2.138 & $\mathrm{~N}$ & -0.510 & -0.375 & -1.892 & $\mathrm{~N}$ \\
\hline \multirow[t]{2}{*}{ Australasia } & Australia & -0.080 & 0.007 & -0.059 & -0.055 & -0.552 & -0.096 & -2.868 & $\mathrm{~N}$ & -0.407 & -0.142 & -2.690 & $\mathrm{~N}$ \\
\hline & New Zealand & -0.031 & 0.007 & 0.049 & 0.046 & -0.734 & -0.051 & -5.032 & $\mathrm{~N}$ & -0.515 & -0.078 & -4.378 & $\mathrm{~N}$ \\
\hline \multirow{2}{*}{$\begin{array}{l}\text { North } \\
\text { America }\end{array}$} & Canada & -0.272 & 0.006 & -0.371 & -0.349 & -0.509 & -0.275 & -0.878 & $\mathrm{~N}$ & -0.347 & -0.260 & 0.013 & $\mathrm{~N}$ \\
\hline & Mexico & -0.338 & 0.005 & -0.449 & -0.424 & -0.649 & -0.407 & -1.480 & $\mathrm{~N}$ & -0.476 & -0.350 & -0.470 & $\mathrm{~N}$ \\
\hline \multirow{3}{*}{$\begin{array}{l}\text { South } \\
\text { America }\end{array}$} & Argentina & -0.030 & 0.001 & -0.261 & -0.033 & -0.033 & -0.261 & 0.013 & $\mathrm{~N}$ & -0.077 & -0.067 & -0.308 & $\mathrm{~N}$ \\
\hline & Brazil & -0.205 & 0.006 & -0.246 & -0.233 & -0.537 & -0.231 & -1.761 & $\mathrm{~N}$ & -0.444 & -0.072 & -1.708 & $\mathrm{~N}$ \\
\hline & Chile & 0.012 & 0.005 & 0.064 & -0.072 & -0.378 & -0.253 & -2.359 & $\mathrm{~N}$ & -0.444 & -0.233 & -2.622 & $\mathrm{~N}$ \\
\hline \multirow[t]{4}{*}{ Europe } & Euro & 0.166 & 0.006 & 0.289 & 0.271 & -0.280 & -0.231 & -2.983 & $\mathrm{~N}$ & -0.103 & -0.001 & -2.712 & $\mathrm{~N}$ \\
\hline & U.K & 0.043 & 0.005 & 0.102 & 0.095 & -0.289 & -0.074 & -2.038 & $\mathrm{~N}$ & -0.190 & -0.150 & -2.045 & $\mathrm{~N}$ \\
\hline & Bahrain & -0.021 & 0.006 & -0.019 & -0.018 & -0.041 & 9.215 & -0.107 & $\mathrm{~N}$ & -0.056 & 6.189 & -0.271 & $\mathrm{~N}$ \\
\hline & Egypt & 0.037 & 0.001 & 0.031 & 0.029 & 0.184 & -0.400 & 0.788 & $\mathrm{~N}$ & 0.249 & -0.496 & 1.603 & $\mathrm{~N}$ \\
\hline \multirow{6}{*}{$\begin{array}{l}\text { Middle } \\
\text { East and } \\
\text { Africa }\end{array}$} & Jordan & -0.026 & 0.001 & -0.034 & -0.031 & -0.181 & -0.560 & -0.763 & $\mathrm{~N}$ & -0.047 & -0.495 & -0.114 & $\mathrm{~N}$ \\
\hline & Kuwait & 0.247 & 0.001 & 0.349 & 0.328 & 0.250 & -0.419 & -0.557 & $\mathrm{~N}$ & 0.240 & -0.338 & -0.681 & $\mathrm{~N}$ \\
\hline & Qatar & 0.037 & 0.000 & 0.041 & 0.038 & 0.289 & -0.500 & 1.308 & $\mathrm{~N}$ & 0.229 & -0.649 & 1.387 & $\mathrm{~N}$ \\
\hline & Saudi Arabia & -0.027 & 0.000 & 0.006 & 0.006 & -0.444 & 0.079 & -2.464 & $\mathrm{~N}$ & -0.249 & 0.025 & -1.852 & $\mathrm{~N}$ \\
\hline & UAE & -0.086 & 0.000 & -0.131 & -0.122 & n.a & -1.000 & n.a & n.a & -0.048 & -0.816 & 0.526 & $\mathrm{~N}$ \\
\hline & South Africa & -0.130 & 0.007 & -0.103 & -0.095 & -0.725 & 0.003 & -2.038 & $\mathrm{~N}$ & -0.545 & -0.041 & -3.676 & $\mathrm{~N}$ \\
\hline Other & Russia & -0.140 & 0.004 & -0.183 & -0.170 & -0.494 & -0.316 & -1.821 & $\mathrm{~N}$ & -0.270 & -0.187 & -0.746 & $\mathrm{~N}$ \\
\hline
\end{tabular}

Note 1: ${ }^{* *}, * * *$ : statistical significance at $10 \%, 5 \%$ and $1 \%$ respectively.
Note 2: The table shows conditional (unadjusted) cross-market correlation coefficients $(\rho)$, adjuster for heteroscedasticity $(\delta)$ and standard deviations $(\sigma)$ for Japan and other stock indexes. The test statistics are obtained from Fisher Z transformations. The stable period is defined as the 12-month pre-earthquake period (March 11, 2010 to March 10, 2011). The short-term turmoil period is defined as the 1-month post-earthquake period (March 11, 2011 to April 10, 2011). The medium-term turmoil period is defined as the 2-month post-earthquake period (March 11, 2011 to May 10, 2011). The full period is the stable period plus the medium-term (March 11, 2011 to April 10, 2011). The medium-term turmoil period is defined as the 2-month post-earthquake period (March 11, 2011 to May 10, 2011). The full period is the stable period plus the medium-term
turmoil period. Co: contagion. While "Y" denotes that the test statistics is greater than the critical value and contagion occurred, "N" indicates that the test statistics was less or equal to the critical value and no contagion occurred. $\rho^{*} \mathrm{stp}, \rho^{*} \mathrm{mtp}$, denote adjusted correlation coefficients for the short and medium term periods respectively. $\delta$ : correlation coefficient adjuster.

Note 3: Correlation coefficients are adjusted for heteroscedasticity using Equation 2. 


\subsubsection{Discussion of results, policy implications and future research directions}

This section has examined if the March 2011 Japanese earthquake, tsunami and nuclear disaster affected the stability of the correlation structure in international stock and foreign exchange markets.

On a first note, with regard to international equity markets, there is substantial evidence of contagion in Taiwan, Bahrain, Saudi Arabia and South Africa. The effect on Saudi Arabia was not unexpected because it is one of the four countries from which a large part of Japan's imports in raw material source. For the other three, cross-market correlations strengthened only with China and Australia in the short-run, although insignificant to account for contagion. A possible explanation as to why Saudi Arabia was most strongly affected both in the immediate and medium terms may be seen from Japan's boost in fuel imports in substitution to energy formerly provided by the wrecked Fukushima nuclear plants. Bahrain, being an oil-export driven economy (like her sisterly neighbor Saudi Arabia), could not have been victim of a different fate. As for Taiwan, as pointed-out by Asongu (2012b), Japan is its second largest trading partner and official estimates on the effect of the Japanese earthquake on the Taiwanese economy stand at a yearly fall in growth by $0.2 \%$ of Gross Domestic Product (GDP).

Secondly, international foreign exchange market results show no presence of contagion. Accordingly, one would have expected the widespread disruption to Japan's US\$5.5 trillion economy to inevitably affect other countries in the Asia-Pacific region and beyond. Regional trade would immediately have been affected by the damage to Japanese ports. The unexpected findings could be explained by the fact that, major Japanese manufacturers of automobiles, semiconductors, computers and other goods immediately took advantage of their international supply chains and production networks. Thus, moving production elsewhere in Asia or to North America, where capacity utilization is still low. Moreover, since Japanese factories generally produce consumer goods rather than intermediate products, disruptions to outbound shipments should not have been expected to seriously affect production processes in other countries.

On managing and mitigating spillovers and contagion, it is worth emphasizing that globalization comes with costs and benefits. Therefore, managing financial market contagion resulting from natural disasters requires that governments minimize the costs and maximize the benefits of financial market integration. Most countries in the sample have undoubtedly benefited from integration, however based on the weight of empirical evidence above; 
measures need to be taken in an effort to manage the downside ramifications of integration in the event of a natural disaster.

The following are some recommendations policy makers need to take into account in order to minimize (mitigate) the adverse financial market effects of disasters. (1) The banking system of a country should not be directly exposed to foreign assets that natural disasters can easily stress and render worthless. This recommendation also applies to assets in institutions that natural disasters could make futile. Accordingly, this caution would attenuate the knockon effects through monetary, financial and real channels. (2) Domestic financial markets (equity, money, foreign exchange and credit markets) may also suffer due to the 'substitution effect'. As credit channels and credit lines in the affected (or contaminated) countries run dry, some of the credit-demand earlier met by overseas financing could easily shift to the domestic sector and put pressure on domestic resources. The reversal of capital flows arising from the de-leveraging process could put pressure on the foreign exchange market, leading to sharp fluctuations in overnight money market rates and undue depreciation of currency. Hence, it is in the interest of central banks to adopt a monetary policy stance that is adequate to growth, inflation and financial stability concerns. (3) In circumstances where the natural disaster mirrors an expected decline in inflation, it is also in the interest of the central bank to adjust its monetary stance and manage liquidity: both domestic and foreign exchange to ensure that credit continues to flow for productive activities at both aggregate and sector specific levels. (4) In order to enable economic agents plan their business activities with more assurance, the central bank could ensure an orderly adjustment of the pain of its policies by maintaining a comfortable liquidity position: ensuring that the weighted average overnight money market rate is maintained within the repo-reverse repo corridor (margin) and ensure conditions conducive for flow of credit to productive sectors (particularly the stressed export industry sectors).

Before we conclude this section, it is important to highlight the implications of this paper to the future of natural disasters. Though the crisis is over, from a financial standpoint the following concerns on future natural disasters are most likely to preoccupy policymakers. (1) Is self-insurance a viable (and/or feasible) option for emerging economies? In other terms, could the accumulation of foreign reserves buffer against financial market crises cropping-up from natural disasters? Whether these reserves derive from current account surpluses (China for example) or capital flows (India for instance), relying on them to hedge contagion could still represent some form of liability. This draws to mind the need of finding a way of balancing the trade-off between 'vulnerability to financial contagion' and 'vulnerability to 
trade contagion' in the event of a disaster. Another imperative strand within this framework points to the redundancy of self-insurance if international arrangements (regional and multilateral) could provide easy, quick and unconditional liquidity during such crises (Asongu, 2012b). (2) How do policy makers keep the financial sector consistent with the real sector in case of a natural disaster? Forgotten and abandoned in the euphoria of financial alchemy is the basic tenet that the financial sector has no standing of its own. Hence, it derives its strength and resilience from the real economic sector. Thinking the other way round has led many into believing that, substantial value could be created by slicing and dicing securities. (3) How should regulatory arbitrage be addressed in times of crisis? If under the nose of regulators, grows a broad, extensive and complex network of a 'shadow banking system' that encourages loose practices, hunt for quick yields and 'non-transparent and risky' financial products, when systems unravel (due to natural disasters), many of these institutions will pose a systematic risk to the financial systems. Therefore, the regulatory architecture has to be modeled (and/or fashioned) to keep pace with innovation and the possibility of natural shocks. (4) Simulating natural disasters and learning how to manage global imbalances resulting from them could also help countries brace for potential financial and real sector consequences of natural crises.

\section{Conclusion}

Financial integration among economies has the benefit of improving allocation efficiency and risk diversification. However the recent global financial crisis, considered as the most severe since the Great Depression has re-ignited a fierce debate about the merits of financial globalization and its implications for growth especially in developing countries. A section in this chapter has examined whether equity markets in emerging countries were vulnerable to contagion during the recent global financial meltdown. Findings have shown: (1) with the exceptions of India and Dhaka, Asian markets were worst hit; (2) but for Peru, Venezuela and Columbia, Latin American countries were least affected; (3) Africa and Middle East emerging markets were averagely contaminated with the exceptions of Kenya, Namibia, Nigeria, Morocco, Dubai, Jordan, Israel, Oman, Saudi Arabia and Lebanon. The results have two important policy implications. Firstly, we have confirmed that Latin America was most prepared to brace the financial crisis, implying their fiscal and monetary policies could serve as a benchmark for other emerging economies. Secondly, we have confirmed that, the strategic opening of the current and capital accounts based on the available weight of 
empirical evidence for a given region/country (as practiced by India) is a caution against global economic and financial shocks.

The effects of natural disasters on financial markets are important in investment decisions, as the benefits of portfolio diversification are severely limited during periods of high volatility and increased cross-market correlations. In the fourth section of the chapter, we have used unadjusted and adjusted correlation coefficients to test for contagion effects across 33 economies in the aftermath of the Japanese earthquake, ensuing tsunami and worst nuclear crisis in recent history. Results have shown that, no international foreign exchange market experienced significantly stronger correlations with the Japanese Yen two months into the crises. However, with respect to international stock markets, Taiwan, Bahrain, Saudi Arabia and South Africa experienced contagion. The results have two paramount implications. Firstly, we have confirmed the existing consensus that in the face of natural crises that could take an international scale, only emerging markets are overwhelmingly affected .Secondly, we have also shown that international financial market transmissions not only occur during financial crisis; natural disaster effects should not be undermined. Other policy implications and future research directions have been discussed.

\section{References}

[1] Anoruo, E., and Mustafa, M. 2007. An empirical investigation into the relation of oil to stock market prices. North American Journal of Finance and Banking Research, 1(1):1-15.

[2] Asongu, S. A. 2011. Political Crises and Risk of Financial Contagion in Developing Countries: Evidence from Africa. Journal of Economics and International Finance, 3(7):462467.

[3] Asongu, S. A. 2012a. Globalization, financial crisis and contagion: time-dynamic evidence from financial markets of developing countries. Journal of Advanced Studies in Finance: Forthcoming.

[4] Asongu, S. A. 2012b. The 2011 Japanese earthquake, tsunami and nuclear crisis: evidence of contagion from international financial markets. Journal of Financial Economic Policy, 4(4):340-353.

[5] Asongu, S. A. 2012c. Are the financial benefits of financial globalization questionable until greater domestic financial development has taken place?. Journal of Economic Studies: Forthcoming.

[6] Calvo, G.A., Leiderman, L., and Reinhart, C.A. 1996. Inflows of capital to developing countries in the 1990s. Journal of Economic Perspectives, 10:123-139. 
[7] Collins. D., and Biekpe, N. 2003. Contagion: a fear for African equity markets? Journal of Economics and Business, 55:285-297.

[8] Coulibaly, B. 2009. Effects of financial autarky and integration: The case of South Africa embargo. Journal of International Money and Finance, 28:454-478.

[9] Demyanyk, Y., and Volosovych, V. 2008. Gains from financial integration in the European Union: Evidence for new and old members. Journal of International Money and Finance, 27:277-294.

[10] Forbes, K. J. 2000. The Asian flu and Russian virus: firm-level evidence on how crises are transmitted internationally. NBER Working Paper 7807.

[11] Forbes, K., and Rigobon, R. 2002. No contagion, only interdependence: measuring stock market co-movements, Journal of Finance, 57(5):2223-2261.

[12] Frankel, J. and Rose, A.K. 1996. Currency crashes in emerging markets: An empirical treatment. Journal of International Economics, 41:351-366.

[13] Henry, P.B. 2007. Capital Account Liberalization: Theory, Evidence and Speculation. Journal of Economic Literature, XLV:887-935.

[14] In, F., Cui, J., and Mahraj, A. 2008. The Impact of a new term auction facility on LIBOR-OIS spreads and volatility transmission between money and mortgage market. http://papers.ssrn.com/sol3/papers.cfm?abstract id=1272806 (unpublished manuscript).

[15] Ji, P. I., and In, F. 2010. The Impact of the global financial crisis on the cross-currency linkage of LIBOR-OIS spreads. Journal of International Financial Markets, Institutions and Money, 20:575-589.

[16] Kanas, A. 1998. Linkages between the US and European equity markets: further evidence from cointegration test. Applied Financial Economics, 8 (6):607-614.

[17] King, M., Sentana, E., and Wadhwani, S.1994. Volatility and links between national stock markets. Econometrica, 62 (4):901-933.

[18] King, M., and Wadhwani, S. 1990. Transmission of volatility between stock markets. Review of Financial Studies, 3(1):5-33.

[19] Kose, M. A., Prasad., E.S., and Taylor, A.D. 2011. Thresholds in the process of international financial integration. Journal of International Money and Finance, 30:147-179.

[20] Lee. H., Wu. H. and Wang. Y. 2007. Contagion effect in financial markets after the South-East Asia Tsunami. Research in International Business and Finance, 21:281-296.

[21] McAndrews, J., Sarkar, A., and Wang, Z. 2008. The Effects of the term auction facility on the London inter-bank offered rate. Federal Reserve Bank of New York Staff Reports No. 335. 
[22] Obstfeld, M. 1986. Rational and self-fulfilling balance of payments crises. American Economic Review, 76:72-81.

[23] Schmukler, S. L. 2004. Financial Globalization: gain and pain for developing countries. Federal Reserve Bank of Atlanta Economic Review: 39-66.

[24] Taylor, J.B., and Williams, J. C. (2008). Further results on a black swan in the money market. Stanford Institute for Economic Policy, Research Discussion Paper 07-046. 